\title{
THE SHAPE OF THE SLOAN DIGITAL SKY SURVEY DATA RELEASE 5 GALAXY POWER SPECTRUM
}

\author{
Will J. Percival, ${ }^{1}$ Robert C. Nichol, ${ }^{1}$ Daniel J. Eisenstein, ${ }^{2}$ Joshua A. Frieman, ${ }^{3,4}$ Masataka Fukugita, ${ }^{5}$ \\ Jon Loveday, ${ }^{6}$ Adrian C. Pope, ${ }^{7,8}$ Donald P. Schneider, ${ }^{9}$ Alex S. Szalay, ${ }^{7}$ Max Tegmark, ${ }^{10}$ \\ Michael S. Vogeley, ${ }^{11}$ David H. Weinberg, ${ }^{12}$ Idit Zehavi, ${ }^{13}$ Neta A. Bahcall, ${ }^{14}$ \\ Jon Brinkmann, ${ }^{15}$ Andrew J. Connolly, ${ }^{16}$ and Avery Meiksin ${ }^{17}$ \\ Received 2006 August 11; accepted 2006 October 1
}

\begin{abstract}
We present a Fourier analysis of the clustering of galaxies in the combined main galaxy and LRG SDSS DR5 sample. The aim of our analysis is to consider how well we can measure the cosmological matter density using the signature of the horizon at matter-radiation equality embedded in the large-scale power spectrum. The new data constrain the power spectrum on scales $100-600 h^{-1} \mathrm{Mpc}$ with significantly higher precision than previous analyses of just the SDSS main galaxies, due to our larger sample and the inclusion of the LRGs. This improvement means that we can now reveal a discrepancy between the shape of the measured power and linear CDM models on scales $0.01 h \mathrm{Mpc}^{-1}<k<$ $0.15 h \mathrm{Mpc}^{-1}$, with linear model fits favoring a lower matter density $\left(\Omega_{M}=0.22 \pm 0.04\right)$ on scales $0.01 h \mathrm{Mpc}^{-1}<$ $k<0.06 h \mathrm{Mpc}^{-1}$ and a higher matter density $\left(\Omega_{M}=0.32 \pm 0.01\right)$ when smaller scales are included, assuming a flat $\Lambda \mathrm{CDM}$ model with $h=0.73$ and $n_{s}=0.96$. This discrepancy could be explained by scale-dependent bias, and by analyzing subsamples of galaxies, we find that the ratio of small-scale to large-scale power increases with galaxy luminosity, so all of the SDSS galaxies cannot trace the same power spectrum shape over $0.01 h \mathrm{Mpc}^{-1}<k<$ $0.2 \mathrm{~h} \mathrm{Mpc}^{-1}$. However, the data are insufficient to clearly show a luminosity-dependent change in the largest scale at which a significant increase in clustering is observed, although they do not rule out such an effect. Significant scaledependent galaxy bias on large scales, which changes with the $r$-band luminosity of the galaxies, could potentially explain differences in our $\Omega_{M}$ estimates and differences previously observed between 2dFGRS and SDSS power spectra and the resulting parameter constraints.
\end{abstract}

Subject headings: cosmological parameters - large-scale structure of universe

\section{INTRODUCTION}

The evolution of perturbations in the early universe imprints characteristic scales that depend on the average matter density (Silk 1968; Peebles \& Yu 1970; Sunyaev \& Zel'dovich 1970; Bond \& Efstathiou 1984, 1987; Holtzman 1989). Fundamentally, the growth of fluctuations is intimately linked to the Jeans scale; perturbations smaller than the Jeans scale do not collapse due to pressure support, while larger perturbations are free to grow

\footnotetext{
1 Institute of Cosmology and Gravitation, University of Portsmouth, Portsmouth, UK.

2 Steward Observatory, University of Arizona, Tucson, AZ.

3 Particle Astrophysics Center, Fermilab, Batavia, IL.

${ }^{4}$ Kavli Institute for Cosmological Physics, Department of Astronomy and Astrophysics, University of Chicago, Chicago, IL.

5 Institute for Cosmic Ray Research, University of Tokyo, Kashiwa, Japan.

6 Astronomy Centre, University of Sussex, Falmer, Brighton, UK.

7 Department of Physics and Astronomy, Johns Hopkins University, Baltimore, MD.

${ }^{8}$ Institute for Astronomy, University of Hawaii, Honolulu, HI.

9 Department of Astronomy and Astrophysics, Pennsylvania State University, University Park, PA.

${ }^{10}$ Department of Physics, Massachusetts Institute of Technology, Cambridge, MA.

11 Department of Physics, Drexel University, Philadelphia, PA.

12 Department of Astronomy, Ohio State University, Columbus, OH.

13 Department of Astronomy, Case Western Reserve University, Cleveland, $\mathrm{OH}$.

14 Department of Astrophysical Sciences, Princeton University, Princeton, NJ.

15 Apache Point Observatory, Sunspot, NM.

16 Department of Physics and Astronomy, University of Pittsburgh, Pittsburgh, PA.

17 SUPA, Institute for Astronomy, University of Edinburgh, Royal Observatory, Blackford Hill, Edinburgh, UK.
}

through gravity. In the radiation-dominated era, the dark matter has negligible density compared to the photon-baryon fluid, and the perturbations in this fluid are stabilized by the high radiation pressure at a time when the sound speed was of order $c / \sqrt{3}$. Consequently, the Jeans scale is of order the horizon scale until matterradiation equality, after which it reduces to zero when the matter dominates. We therefore see that the horizon scale at matterradiation equality will be imprinted in the distribution of fluctuations: this scale marks a turnover in the growth rate of fluctuations.

In a model with only collisionless dark matter, all lengths scale with the horizon scale at matter-radiation equality, which is a multiple of $\left(\Omega_{M} h^{2}\right)^{-1}$, where $h=H_{0} / 100 \mathrm{~km} \mathrm{~s}^{-1} \mathrm{Mpc}^{-1}$. Consequently, cold dark matter (CDM) transfer function fitting formulae (such as eq. [G3] of Bardeen et al. 1986) were traditionally created as a function of $q \equiv k /\left(\Omega_{M} h^{2} \mathrm{Mpc}\right)$. When analyzing galaxy redshift surveys, the comoving distance-redshift relation introduces another factor of $h$ into the measurements, so the data constrain the transfer function in $k /\left(h \mathrm{Mpc}^{-1}\right)$. Consequently, fits between model and data constrain the degenerate parameter combination $\Omega_{M} h$.

Such cosmological constraints are important for breaking degeneracies between cosmological parameters that exist when fitting just cosmic microwave background (CMB) data (Eisenstein et al. 1999; Efstathiou \& Bond 1999; Percival et al. 2002). With increasingly precise temperature and polarization $\mathrm{CMB}$ constraints (Hinshaw et al. 2006; Page et al. 2006; Spergel et al. 2006), the additional cosmological role of galaxy surveys to provide cross-checks is becoming increasingly important. In this paper we carefully test the methodology behind using the shape of the galaxy power spectrum to provide cosmological constraints. 
Extracting the cosmological information encoded in the galaxy power spectrum has motivated many previous surveys. Early studies, with of order $10^{4}$ galaxies, include the CfA (Vogeley et al. 1992; Park et al. 1994), LCRS (Shectman et al. 1996), and PSCz (Saunders et al. 2000) surveys. These surveys were able to show that the shape of the power spectrum required a relatively small matter density, at odds with the simple Einstein-de Sitter model (e.g., Efstathiou et al. 1990; Tadros et al. 1999). The same general shape of the power spectrum has been recovered by deprojecting the APM (Maddox et al. 1990, 1996) survey, the parent catalog of the Two Degree Field Galaxy Redshift Survey (2dFGRS; Efstathiou \& Moody 2001; Padilla \& Baugh 2003), and by deprojecting the photometric component of the Sloan Digital Sky Survey (SDSS) catalog (Scranton et al. 2002; Connolly et al. 2002; Tegmark et al. 2002; Dodelson et al. 2002; Szalay et al. 2003). More recently, photometric redshifts have been exploited to improve the deprojection of galaxy distances and the accuracy with which the power spectrum can be measured from photometric data. Two studies have recently been completed considering luminous red galaxies (LRGs) within the SDSS (Padmanabhan et al. 2006; Blake et al. 2007), showing a marked improvement in accuracy on previous work.

However, the most precise measurement of the three-dimensional galaxy power spectrum is still recovered from redshift surveys. Over the past 5 years there has been a revolution in the number of galaxy redshifts measured and the size of the volume probed by these surveys. Leading the way have been two large galaxy redshift surveys, the 2dFGRS (Colless et al. 2001, 2003) and SDSS (York et al. 2000). In this paper we analyze the relative clustering strengths of galaxy samples as a function of scale. The overall clustering amplitude is also potentially interesting in constraining cosmological models but takes more work to decode. This normalization is known to be a strong function of both galaxy color and luminosity (Park et al. 1994; Norberg et al. 2001, 2002; Zehavi et al. 2002), so an understanding of galaxy bias is required before cosmological constraints can be derived from this statistic. However, the effect of galaxy bias on the shape of the power spectrum is less certain and is the subject of this paper. On small scales, where nonlinear corrections to the matter power spectrum are important, the shapes of galaxy power spectra are known to depend on galaxy color and luminosity (e.g., Cole et al. 2005). However, on large scales, the effects on the shape are more uncertain.

On larger scales where the matter in the universe is still expected to be predominantly in the linear regime $k \lesssim 0.15 h \mathrm{Mpc}^{-1}$ (Smith et al. 2003), discrepancies currently exist between the shapes of the power spectra recovered from the $2 \mathrm{dFGRS}$ and SDSS. The problem is demonstrated by the measurements of $\Omega_{M}$ obtained from such fits. Assuming a Hubble parameter $h=0.72$, the Tegmark et al. (2004) SDSS main galaxy analysis favored $\Omega_{M}=0.296 \pm 0.032$. Similar values of $\Omega_{M} \simeq 0.3$ were found by alternative analyses of the red-selected SDSS main galaxy power spectrum (Pope et al. 2004), a set of SDSS spectroscopic LRGs (Eisenstein et al. 2005), and SDSS photometrically selected LRGs (Padmanabhan et al. 2006; Blake et al. 2007). In combination with the Wilkinson Microwave Anisotropy Probe (WMAP) 1 yr data (Spergel et al. 2003), the Eisenstein et al. (2005) analysis of the LRGs provided the constraint $\Omega_{M}=0.273 \pm 0.025$ from a combination of the overall shape of the correlation function and the peak caused by baryon acoustic oscillations. In contrast, a lower matter density $\Omega_{M}=0.231 \pm 0.021$ is favored by measurements of clustering of blue-selected galaxies in the 2dFGRS by Cole et al. (2005), who included a simple model for scaledependent bias, although this had a relatively minor effect on the recovered matter density. Recent results from the 3 yr $W M A P$ data have provided an independent constraint on the matter density, finding $\Omega_{M}=0.234 \pm 0.035$ (mean constraint from Table 2 of Spergel et al. 2006) from a better resolution of the third peak height. It is clear that these discrepancies between measurements are not linked to a single technique or particular analysis.

When the constraints from the galaxy power spectra are combined with the CMB data, the discrepancy is still clear. From Table 5 of Spergel et al. (2006) we see that combining the $3 \mathrm{yr}$ $W M A P$ data with additional cosmological constraints from the $2 \mathrm{dFGRS}$ power spectrum of Cole et al. (2005) gives $\Omega_{M}=$ $0.236_{-0.029}^{+0.016}$. However, when the $W M A P$ data are combined with constraints from the SDSS power spectrum of Tegmark et al. (2004), the higher $\Omega_{M} h$ constraint from the large-scale structure data increases the best fit to $\Omega_{M}=0.266_{-0.040}^{+0.025}$ (see Table 6 of Spergel et al. 2006).

The tension between measurements from different large-scale structure experiments and the CMB observations is at the level of approximately $2 \sigma$, and it is therefore possible that it could be explained by cosmic variance. Alternative explanations include a scale-dependent galaxy bias on scales $k \lesssim 0.15 h \mathrm{Mpc}^{-1}$, or a systematic problem with one of the data sets. In this paper we test these possibilities by measuring the redshift-space power spectrum from the latest SDSS sample, Data Release 5 (DR5), which contains approximately twice as many galaxies as previously analyzed and 60\% more LRGs than used in Eisenstein et al. (2005). By optimally analyzing all of the galaxies to calculate the underlying power spectrum, we obtain the most accurate determination of the redshift-space power spectrum ever obtained for any sample of galaxies. First, we test if the discrepancy between previous $2 \mathrm{dFGRS}$ and SDSS power spectra remains. Second, the size of the sample means that we can consider how well the galaxies trace the mass, by testing the simple hypothesis that the shape of the power spectrum matches linear CDM models over $k<0.15 h \mathrm{Mpc}^{-1}$, and looking for discrepancies over these scales. Finally, the number of galaxies and volume covered are now sufficient to accurately measure power spectra for subsamples, splitting the catalog to test for general changes in the shape of the power spectrum as a function of galaxy properties.

In a parallel paper, Tegmark et al. (2006) present an analysis of a largely overlapping data set, drawn from SDSS DR4, with a focus on the implications for multiparameter cosmological model fits. There are a number of differences in the analysis methods. Tegmark et al. (2006) use a pseudo-Karhoenen-Loeve method (Vogeley \& Szalay 1996; Tegmark et al. 1997) to estimate the real-space galaxy power spectrum, using finger-of-God compression and linear theory to remove redshift-space distortion effects. We use the Fourier method of Percival et al. (2004), which extends that of Feldman et al. (1994) to estimate the angle-averaged (monopole) redshift-space galaxy power spectrum. We combine the LRG and main galaxy samples, while Tegmark et al. (2006) concentrate on the LRGs, after showing that they have a power spectrum shape consistent with that of the main sample. In addition, the many technical decisions that go into these analyses, regarding completeness corrections, angular masks, $K$-corrections, and so forth, were made independently for the two papers, and they present different tests for systematic uncertainties. Despite these many differences of detail, our conclusions agree to the extent that they overlap (this will be discussed in Tegmark et al. 2006), a reassuring indication of the robustness of the results.

\section{THE SDSS DR5 SAMPLE}

SDSS (York et al. 2000; Stoughton et al. 2002; Abazajian et al. 2003, 2004; Adelman-McCarthy et al. 2006) is an ongoing survey using a $2.5 \mathrm{~m}$ telescope (Gunn et al. 2006) to obtain $10^{4} \mathrm{deg}^{2}$ of 
imaging data in five passbands $u, g, r, i$, and $z$ (Fukugita et al. 1996; Gunn et al. 1998). The images are reduced (Lupton et al. 2001; Stoughton et al. 2002; Pier et al. 2003; Ivezic et al. 2004) and calibrated (Lupton et al. 1999; Hogg et al. 2001; Smith et al. 2002; Tucker et al. 2006), and galaxies are selected in two ways for follow-up spectroscopy. The main galaxy sample (Strauss et al. 2002) targets galaxies brighter than $r=17.77$ (approximately $90 \mathrm{deg}^{-2}$, with a median redshift $z=0.11$ ); in this paper we use the DR5 sample (J. Adelman-McCarthy et al. 2007, in preparation) containing 465,789 main galaxies that meet our selection criteria. In a small subset of the data taken during initial survey operation, we set a conservative faint magnitude limit corresponding to $r=17.5$, to avoid minor fluctuations in the survey depth.

In addition to the main galaxy sample, we also select 56,491 cut I and cut II LRGs (Eisenstein et al. 2001). The selection of these galaxies, based on $g, r$, and $i$ colors and going to a deeper $r$ magnitude, adds approximately 15 galaxies $\mathrm{deg}^{-2}$ in addition to the main sample and extends the redshift distribution to $z \simeq 0.5$. In our sample, 65,032 of the main galaxies are also targeted in the SDSS as LRGs, but only 21,310 of these galaxies are intrinsically luminous with $M_{0.1_{r}}<-21.8\left(M_{0.1_{r}}\right.$ is defined in $\left.\S 2.3\right)$. Our sample therefore consists of 77,801 bright LRGs and 444,479 other galaxies, giving 522,280 galaxies in total.

These galaxies and objects selected for SDSS spectroscopic observation for other scientific programs are assigned to plug plates using a tiling algorithm designed to ensure nearly complete samples (Blanton et al. 2003a). The spectra are good enough to allow redshifts to be obtained for almost all of the galaxies selected for observation. A detailed review of the SDSS galaxy observing strategy and the main galaxy sample is given by Tegmark et al. (2004).

In our investigation, we simultaneously analyze the main galaxy sample and the LRGs, therefore including correlations between the two data sets in addition to internal correlations within the individual subsamples. This combination is aided by the fact that the transition from main galaxies to LRGs within the survey is smooth in terms of galaxy properties and expected bias. In this section we present an overview of the data and the techniques used to model the selection function of this combined sample.

Our chosen analysis method uses an empirically determined model of luminosity-dependent (but scale-independent) bias to increase the accuracy with which the underlying power spectrum can be recovered and correct for offsets in the measured power caused by such a bias (a description of the model is provided in $\S 3)$. We apply two cuts to the final DR5 subsample that we analyze as a consequence of this model: we exclude low-luminosity LRGs with $M_{0.1_{r}}>-21.8$ and high-luminosity galaxies with $M_{0.1_{r}}<-23.0$ (whether main galaxy or LRG). The reasons for these cuts are presented in $\S 3$.

Due to practical limitations of fiber positioning, spectra cannot be obtained for objects closer than 55", within a single spectroscopic tile. This is mitigated to some extent by multiple observations of the same region where tiles overlap, but we choose to apply a further correction; if a targeted main galaxy or LRG has no redshift and lies within $55^{\prime \prime}$ of another main galaxy or LRG with a redshift, then the observed redshift is assigned to both galaxies. Main galaxies (non-LRGs) and LRGs are treated separately; where a main galaxy or LRG is not assigned a fiber due to collision with a quasar or galaxy that is not in the same class (e.g., a main galaxy is obscured by an LRG), then no redshift is assigned. This is the procedure adopted by Zehavi et al. (2002) where, by comparison with just the plate overlap regions where fiber collision has a reduced impact, it was shown to provide a sufficient correction for this effect on the large scales of interest in the current study. We apply a close-pair correction to 3132 LRGs and 19,402 main galaxies, which form $4.3 \%$ of the total population.

In certain regions observed early in the survey operations, we exclude all main galaxies with $r>17.5$, so the main galaxies form two populations with $r<17.5$ or $r<17.77$ depending on angular position. A total of 49,688 of the main sample galaxies $(11.9 \%)$ lie in the regions limited to $r<17.5$. We assume that the LRGs form a single population with an isotropic redshift distribution. The radial selection function of our combined sample can therefore be decomposed into three subsamples with different radial distributions: main galaxies with $r<17.5$, main galaxies with $r<17.77$, and LRGs. The limits of the survey are trimmed by setting redshift limits on the combined sample by only considering galaxies with $0.003<z<0.5$. LRGs not in the main galaxy sample must have $z>0.15$, and main sample galaxies must have $z<$ 0.3 . This removes regions where the selection function becomes small and poorly determined.

\subsection{Angular Selection}

The angular selection function of the SDSS galaxies was modeled using a routine based on a HEALPix (Górski et al. 2005) equal-area pixelization of the sphere. HEALPix was used to decompose the sphere into 3,145,728 pixels, each of size $0.013 \mathrm{deg}^{2}$. This means that each SDSS plate is covered by 532 pixels, and the DR5 sample analyzed covers 487,177 pixels. Given the large angular scales of interest, the effect of this pixelization should be negligible on the resulting power spectrum.

The first task is to find groups of pixels that have the same spectroscopic targeting information: they cover regions selected in the same targeting run(s), covered by the same set of tiles, and that are within the photometric SDSS region, i.e., not in a bad field. The SDSS photometric survey area is decomposed into fields of approximate size $0.033 \mathrm{deg}^{2}$. Consequently, bad fields, as defined in the SDSS Catalogue Archive, often only cover part of a pixel, so for each pixel we have allowed the effective area to be reduced by bad fields rather than removing the whole pixel. There are small internal regions within the area covered by the SDSS imaging that are not covered by spectroscopic tiles, which only have a few possible target galaxies, if any at all. We have included such regions as separate groups of pixels in our analysis. In total, we decompose the survey region in 6447 distinct groups. Note that we use a full list of targeted plates to create this decomposition of the spherical surface rather than just the observed plates; it is the targeting algorithm (Blanton et al. 2003a) that decides the distribution of the spectroscopically observed galaxies, so the distribution across observed plates will depend on the positions of the unobserved overlapping plates.

An example region within the angular mask created for the survey is plotted in Figure 1, showing the different groups. The staves (named for the similarly shaped planks in barrel making) within the survey, corresponding to photometric sections from different great circle scans, are clearly visible. The most obvious pattern in the decomposition of the angular mask is due to the overlap of different spectroscopic plates, which can leave some small regions containing only a handful of galaxies. The boundaries to different targeting regions follow segments of staves, leading to additional divisions across different staves. This region was chosen as it includes a small area covered by bad fields that shows the interleaved drift-scan strategy of the SDSS photometric observations, splitting the stave into 12 individual columns.

Having created a list of target regions on our pixelated mask, we calculated the completeness within each group, the ratio of 


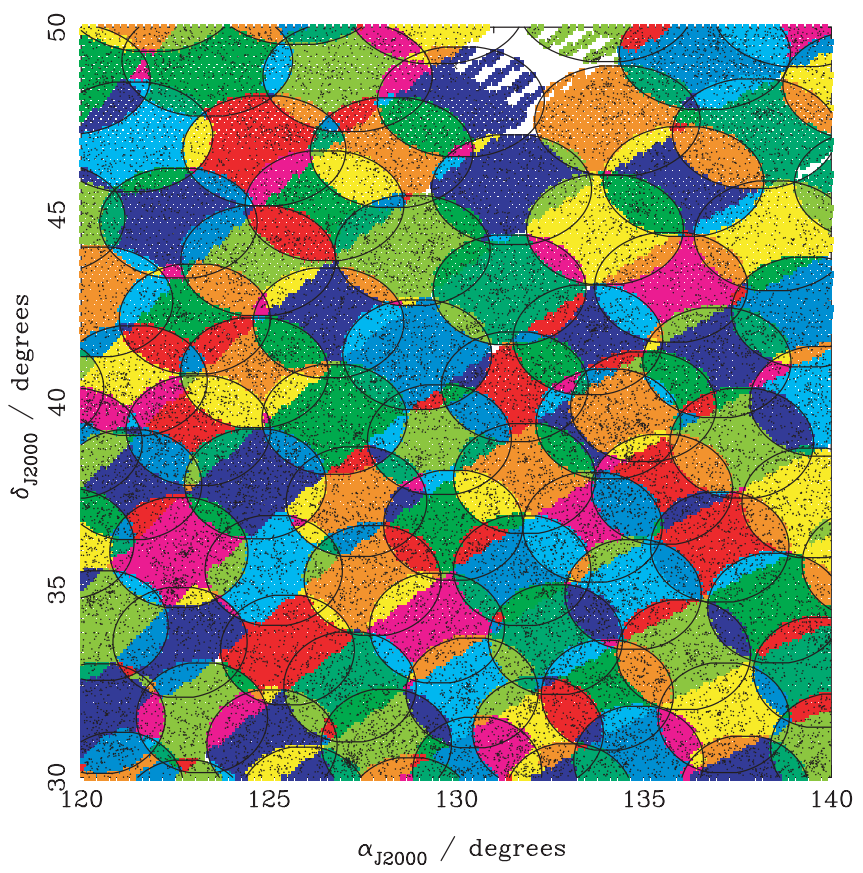

FIG. 1.-Part of the SDSS DR5 sample region plotted as a function of celestial coordinates. Note that this is not an equal-area projection, so the plate outlines (solid lines) do not form perfect circles. Different colors delineate groups of pixels where the completeness of the survey is expected to be the same (see text for details). Black points show the positions of the galaxies. White regions are bad areas excluded from the survey mask. Where these areas are due to bad photometric fields, the regions often follow the drift scanning strategy of the photometric survey, hence the white stripes at $\alpha \simeq 132^{\circ}, \delta \simeq 48^{\circ}$.

good-quality spectra to targets. Any small internal group containing no targets was assigned a completeness of 1 . We have applied a completeness cut of $70 \%$ and exclude regions with a lower completeness. In general, this only removes regions where the spectroscopic survey is incomplete and further observations are scheduled for a particular group. For our final sample, 97.6\% of the galaxies targeted have redshifts with spectroscopic confidence greater than $80 \%$, as defined in the SDSS Catalogue Archive Server (CAS), after the fiber collision correction mentioned above.

\subsection{Radial Selection}

In order to fully quantify the expected distribution of galaxies, we obviously need to model the radial galaxy distribution. We do this in different ways for the main galaxies and LRGs. For the main galaxies, we have found that a simple fit of the form (Baugh \& Efstathiou 1993)

$$
f(z)=z^{g} \exp \left[-\left(\frac{z}{z_{c}}\right)^{b}\right]
$$

provides an adequate fit to the data, where $g, b$, and $z_{c}$ are fitted parameters. Figure 2 shows the distribution of main galaxy redshifts in the SDSS DR5 sample compared with a fit of this form for apparent magnitude limits of $r=17.5$ and 17.77; as discussed in $\S 2.1$, in some angular directions the effective magnitude limit had to be reduced to $r=17.5$. For galaxies with $r<17.5$, the best-fit model redshift distribution has $z_{c}=0.0955, b=1.88$, $g=1.35$. For galaxies with $r<17.77$, the best-fit value of $z_{c}$ changes to $z_{c}=0.106$.

For the LRGs, we fit the radial number density using a cubic spline (Press et al. 1992) with nodes separated by $\Delta z=0.05$,

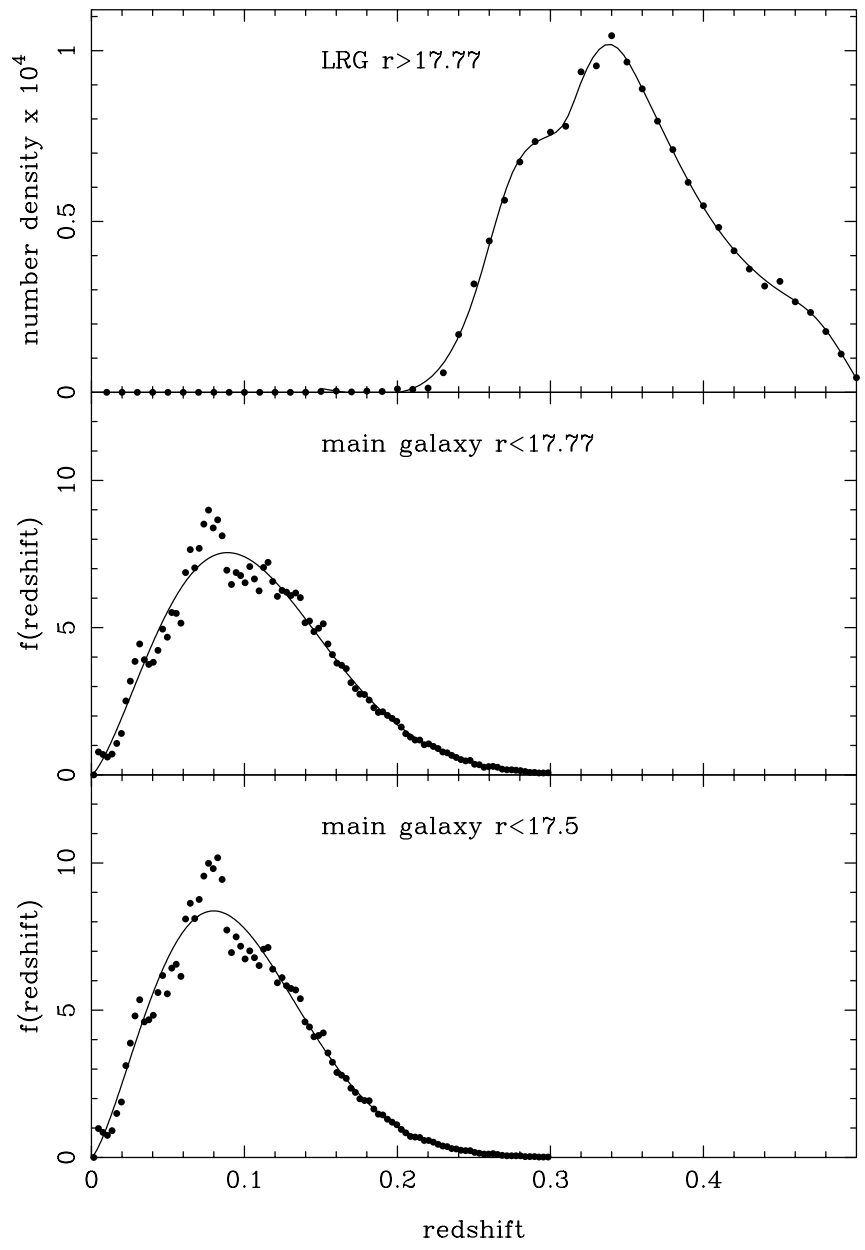

FIG. 2.-Radial distributions of the SDSS DR5 main galaxies ( filled circles) to an apparent $r$ magnitude limit of 17.5 (bottom) and 17.77 (middle). The sharp increase in the number of galaxies at $z=0.08$ is predominantly the effect of the "Sloan Great Wall" (Gott et al. 2005). These data are fitted using eq. (1), shown by the solid lines (Baugh \& Efstathiou 1993). In the top panel we show the distribution of the number density of the LRGs (excluding those in the main galaxy sample). These data are fitted with a smooth cubic spline (solid lines) with nodes selected to allow the curve to fit the sharp distortions caused by spectroscopic features moving through the SDSS filters used to select the LRGs and the mixture of cut I and cut II LRGs (see text for details).

although we add additional nodes to enable the fit to match the distortions at $z \simeq 0.3,0.34$, and 0.44 , caused by spectroscopic features moving through the SDSS filters used to select the LRGs, and the join between cut I and cut II LRGs. At the higher redshifts probed by the LRGs, the effect of clustering on the redshift distribution diminishes, and a spline fit is less likely to remove structure compared with lower redshift data.

\subsection{Luminosities}

Where specified, we have $K$-corrected the galaxy luminosities using the methodology outlined in Blanton et al. (2003a, 2003b). In particular, we have used the kcorrect_v4_1_4 software package using the observed $u, g, r, i, z$ Galactic extinction-corrected (using the maps of Schlegel et al. 1998) Petrosian magnitudes from the DR5 CAS archive and their measured errors. We have used the "BEST" database to obtain the galaxy magnitudes and the "TARGET" database to obtain the list of galaxy targets. We also use the same $z=0.1$ shifted $r$-band filter to define our luminosities (as discussed in Blanton et al. 2003b), which we refer to as $M_{0.1}$ throughout this paper. Galaxy luminosities without $K$-corrections are written as $M_{r}$. We use the same method for both 


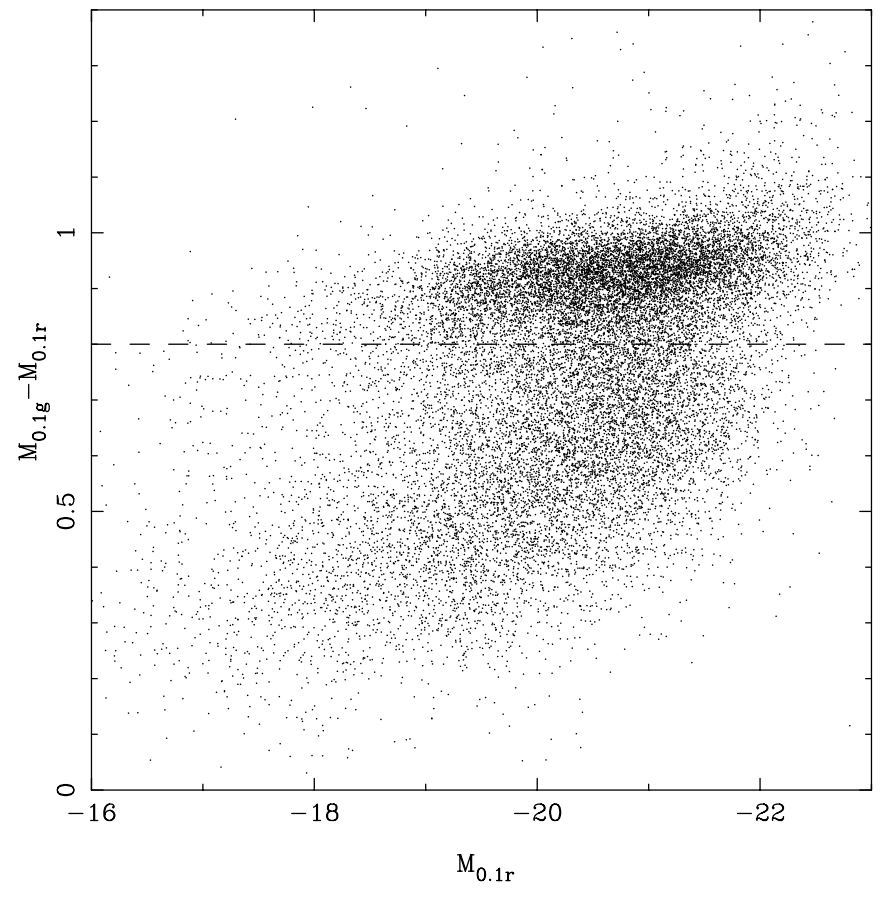

FIG. 3.-Color of 5\% of the main galaxies in the SDSS DR5 sample, selected at random. The well-known bimodal split between red and blue galaxies is clear, approximately split by $M_{0.1_{g}}-M_{0.1_{r}}=0.8$ (dashed line). This plot highlights the fact that as we change the magnitude of the samples, we also change the average color, with the more luminous main galaxies being predominantly redder.

the LRG sample and the main SDSS galaxy sample and have not added evolutionary corrections. We have assumed $\Omega_{M}=0.3$ and $\Omega_{\Lambda}=0.7$ for computing these $K$-corrections and applied recommended AB corrections to the observed SDSS magnitude system (Smith et al. 2002).

\subsection{Colors}

The average colors of galaxies change as the luminosity increases. Figure 3 shows the $M_{0.1_{g}}-M_{0.1_{r}}$ color distribution of these galaxies, plotted as a function of $M_{0.1}$. The well-known bimodal red-blue split in galaxy colors (Strateva et al. 2001; Baldry et al. 2004 ) is evident and can be approximately delineated by $M_{0.1_{g}}-$ $M_{0.1_{r}}=0.8$. Importantly, for our development of a bias model for these galaxies, as we change the magnitude we also change the color of our sample, with more luminous galaxies being predominantly redder. We see in the next section that this means that there is a smooth transition between the main galaxy and LRG samples.

\section{MODELING SCALE-INDEPENDENT GALAXY BIAS}

For a magnitude-limited survey, on average, the largest scales will be probed by the most luminous galaxies. Luminous galaxies are known to be more biased than less luminous galaxies (e.g., Park et al. 1994), so this relative bias needs to be quantified in order to minimize any systematic effects caused by comparing different types of galaxies on different scales. For the main galaxy sample we analyze subsamples with different average luminosity, in order to measure the relative bias ( $\S 3.1)$. The LRG sample is now sufficiently large that we can also consider splitting this catalog into subsamples as a function of luminosity (this is discussed in $\oint 3.2$ ).

\subsection{Main Galaxies}

In order to empirically determine the relative clustering strengths of the main galaxy sample as a function of luminosity,

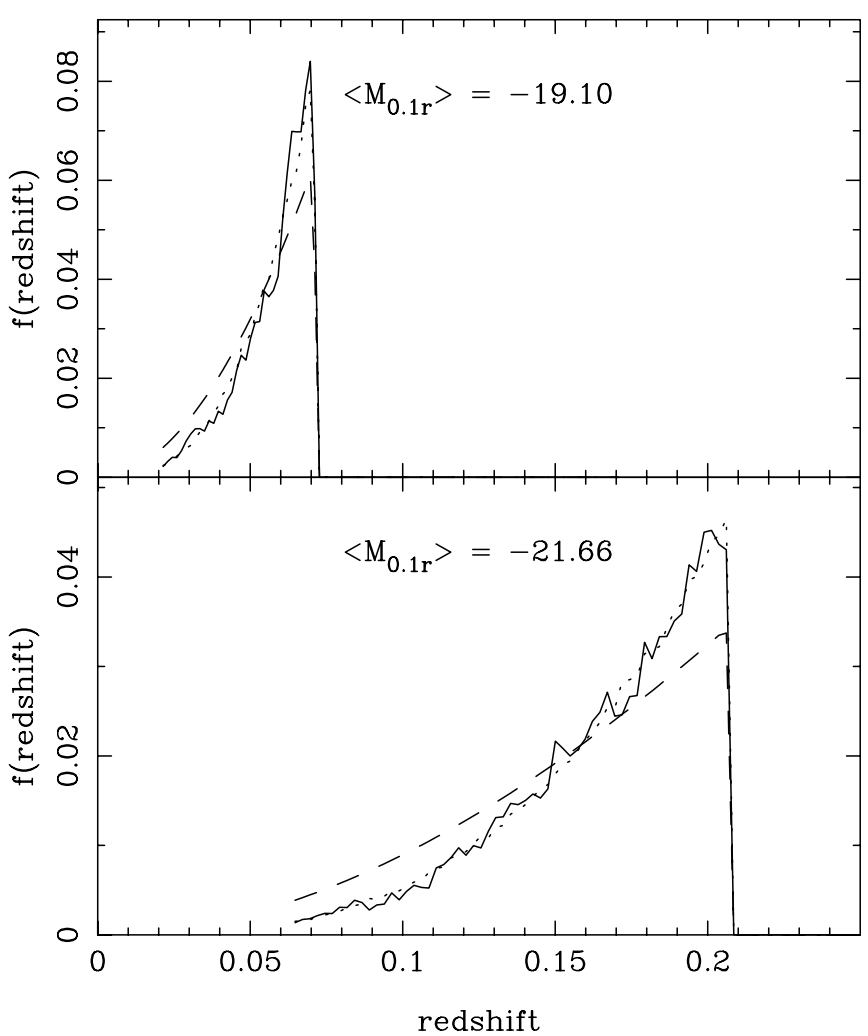

FIG. 4.-Redshift distributions of two of our pseudo-volume-limited catalogs (solid lines). The selection of these catalogs was based on absolute magnitudes without $K$-corrections or corrections for evolution and so are not strictly volume limited. These effects were instead included in estimating the redshift distribution of the samples to create matched random catalogs (dotted lines; see text for details). For comparison, the dashed lines show the redshift distribution that the catalogs would have if they were strictly volume limited: as can be seen, these are a poor fit to the data.

we split the catalog based on the absolute magnitude without $K$-correction or correction for evolution in the luminosity function and the corresponding redshift limits where the subcatalog is complete (see Fig. 5). We analyze eight catalogs of width $\Delta M_{r}=$ 0.5 with $-22.0 \leq M_{r} \leq-18.0$ and an additional catalog of bright galaxies with $-\overline{23}<\bar{M}_{r}<-22$. We call these catalogs pseudovolume-limited, as they are not strictly volume limited because we have ignored $K$-corrections and evolution in the luminosity function. In order to estimate the redshift distribution of each sample, we determine the average $K$-correction as a function of redshift and use this, together with the best-fit evolutionary corrections of Blanton et al. (2003b), to predict the expected galaxy number density. The redshift distributions for two of our pseudo- volumelimited catalogs are compared with the modeled distribution in Figure 4, where good agreement is demonstrated. Obviously, we can still calculate the average absolute magnitude including $K$-corrections for each sample, and it is this magnitude that we use to parameterize our bias model.

The distribution of absolute magnitude (without $K$-correction) against redshift for the SDSS DR5 sample of galaxies is presented in Figure 5. We plot $M_{r}$ against redshift for 5\% of the galaxies in the combined sample, randomly selected. The main galaxy volumelimited catalogs are delineated by the boxes in this plot, and the upper and lower apparent magnitude limits of the main galaxy survey are also shown.

For each pseudo-volume-limited catalog we have calculated the power spectrum using the same method applied to our final catalog in $\S 4$, with the exception that a uniform bias model was 


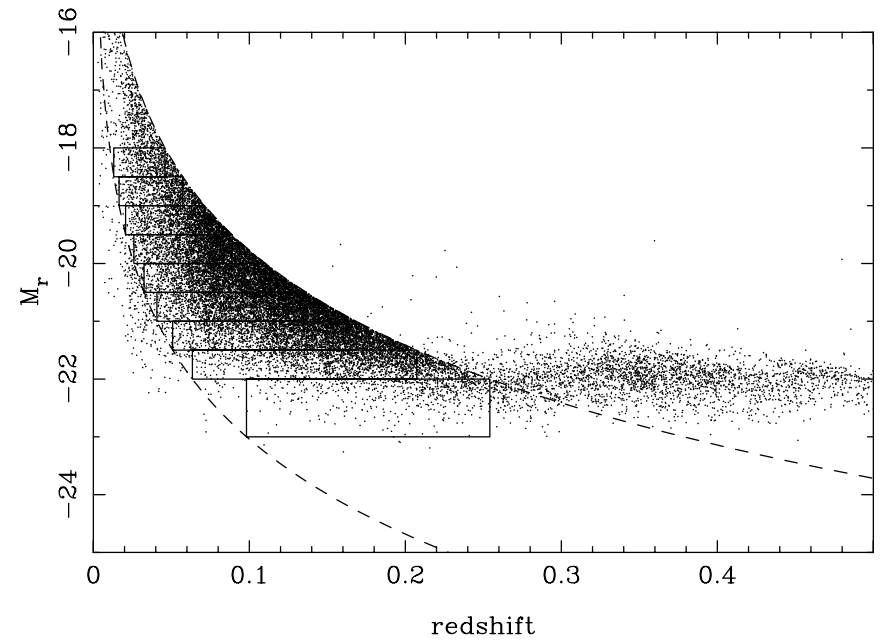

FIG. 5.-Distribution of the SDSS galaxies in the redshift-luminosity plane. Absolute magnitudes were calculated assuming a flat $\Lambda$ cosmology with $\Omega_{M}=$ 0.3 and have not been $K$-corrected or corrected for evolution. The upper and lower apparent magnitude limits of the main galaxy sample are shown by the dashed lines. The redshift and magnitude limits of the pseudo-volume-limited catalogs analyzed to calculate the relative bias as a function of absolute magnitude are shown by the overlaid rectangles.

applied: we treat all galaxies as having $b=1$, so the relative amplitudes of the recovered power are not affected by an input bias model. Some of these power spectra are plotted in Figure 18. The window function for each subcatalog was calculated as for our final catalog, and we have fitted the power spectrum amplitude over $0.01 h \mathrm{Mpc}^{-1}<k<0.2 h \mathrm{Mpc}^{-1}$ using window-convolved models with approximately the correct large-scale shape. The power spectra recovered from the different subsamples are correlated, and we have not estimated their relative errors, as would be strictly required when comparing their relative amplitudes. Instead, we simply measure the average and standard deviation of the difference between measured and model power spectra over the range of scales of interest and calculate the bias from this. Consequently, the biases are not optimally determined, and their errors do not include the effect of cosmic variance for the regions of the catalogs that do not overlap and will probably therefore underestimate the true error. However, as we show in test 5 of $\S 7$, we do not need to know the relative biases to high precision as the resulting power spectrum is not sensitive to the exact form of this correction.

The relative biases measured from the pseudo-volume-limited catalogs are plotted (filled circles) in Figure 6 as a function of the average $K$-corrected absolute magnitude. The smooth shape matches the overall shape of the simple formula of Norberg et al. (2001), $b / b_{*}=0.85+0.15 L / L_{*}$ (solid line), with $M_{*}=-20.44$ (Blanton et al. 2003b). However, the addition of an extra term, $b / b_{*}=0.85+0.15 L / L_{*}+0.04\left(M_{*}-M_{0.1_{r}}\right)$ ( Tegmark et al. 2004; Zehavi et al. 2005b), allows for a sharper increase in bias with luminosity, which is a better fit to the data.

When measuring the power spectrum from the final combined galaxy catalog (see $\S 4$ for a description of the method), we only need to know the averaged properties of the expected bias at each spatial location. Consequently, even though we expect galaxy bias to depend on color as well as luminosity, a simple luminosity-bias relation can still be used provided that the catalogs from which the relation is derived contain the same distribution of galaxy colors in each luminosity bin as galaxies of that luminosity in the combined galaxy sample. For our pseudo-volume-limited subcatalogs of the main sample galaxies, we only exclude galaxies that lie beyond the redshift limits applied for each catalog (see Fig. 5). Because

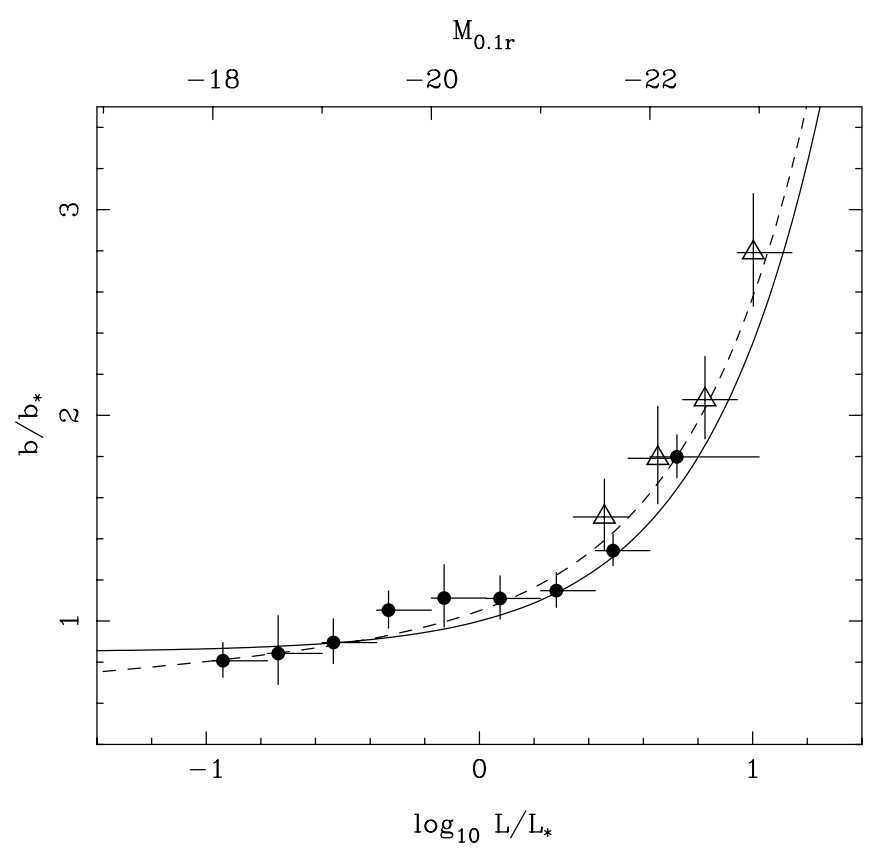

FIG. 6.-Bias of the SDSS main galaxies ( filled circles with $1 \sigma$ errors) and LRGs (open triangles with $1 \sigma$ errors) as a function of $M_{0.1}$. Horizontal errors show the range of luminosities analyzed in each sample (ignoring $K$-corrections), while the data point is placed at the weighted average $K$-corrected luminosity. The relation suggested by Norberg et al. (2001), $b / b_{*}=0.85+0.15 L / L_{*}$, where $M_{*}=$ -20.44 (Blanton et al. 2003b), is shown by the solid line. The dashed line shows the alternative formula of Tegmark et al. (2004) predicting a steeper increase in bias for luminous galaxies. As we are only interested in the relative normalization of the bias, we allow $b_{*}$ to vary to fit the data for the different formulae, meaning that the lines do not cross at $L=L_{*}$.

the catalogs are relatively narrow in magnitude, $\Delta M_{r}=0.5$, they contain between $67 \%$ and $77 \%$ of the total number of galaxies within the chosen magnitude limits (this number fluctuates because of the changing steepness of the number counts shown in Fig. 5). Consequently, each subcatalog should have approximately the same galaxy color distribution as all galaxies of that luminosity in the main sample. At high luminosities, the galaxies are predominantly LRGs as shown in Figure 3, while at low luminosities they are formed of a mix of red and blue galaxies.

\section{2. $L R G S$}

The DR5 LRG sample is of sufficient size that the clustering can now be measured as a function of luminosity as for the main galaxy sample. The distribution of LRG magnitudes is plotted against redshift in Figure 5, showing that the LRGs form a natural extension of the main galaxy sample to higher redshifts. However, given the more complicated selection function of the LRGs, modeling the redshift distribution is not as straightforward as for the main galaxy sample. Consequently, for subcatalogs of LRGs selected as a function of luminosity, we have fitted the number density as a function of redshift with the cubic spline fit as described in $\S 2.2$ for the total LRG sample. Example fits are plotted for two of our LRG subcatalogs in Figure 7. Because of the increased volume covered by the LRGs, although there are relatively few galaxies, the effect of cosmological structure is small, and we do not expect to remove power by fitting to the redshift distribution in this way.

The relative bias measurements for the LRGs were calculated from these subsamples as for our main galaxy pseudo-volumelimited catalogs and are plotted in Figure 6 compared with the main galaxy sample measurements. As can be seen, the bias increases 


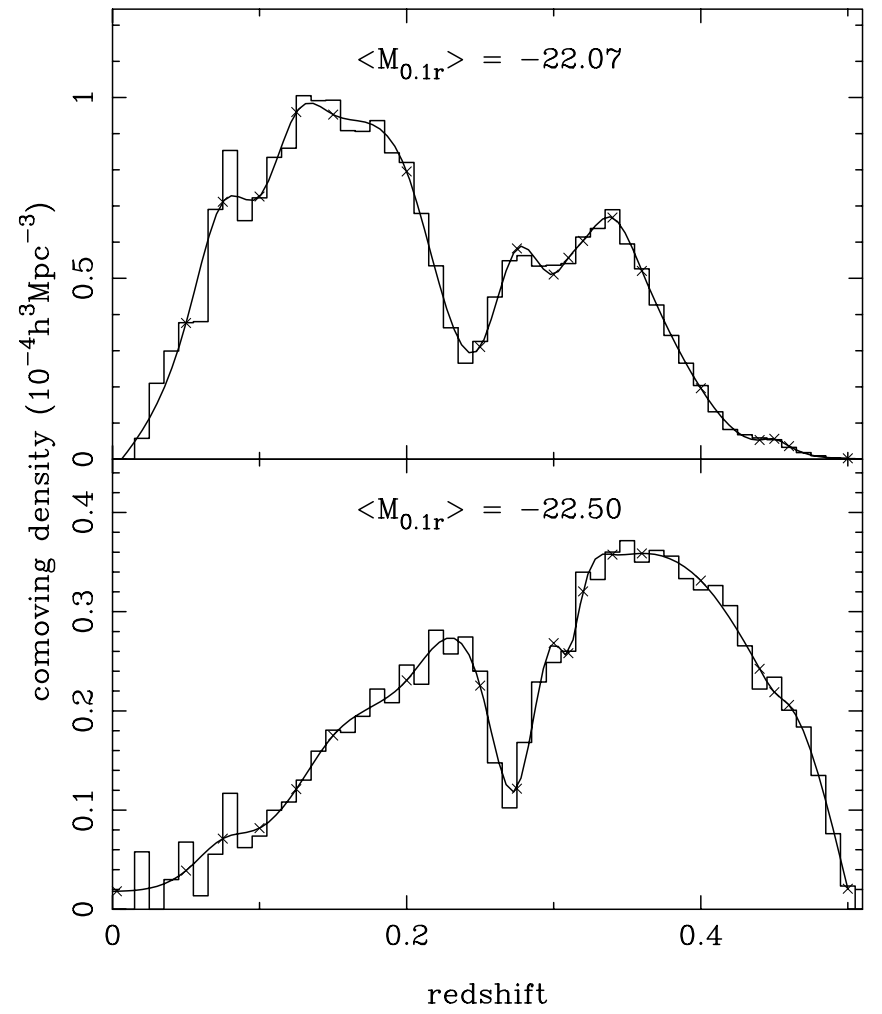

FIG. 7.-Number density as a function of redshift of two of our magnitudeselected LRG catalogs (solid histograms). The distribution is fitted by a cubic spline (smooth curve) with the positions of the nodes (crosses) determined as described in the text.

with luminosity for the LRGs as for the main galaxies, and the high-luminosity data match the simple formula of Tegmark et al. (2004). We do see a difference between LRG and main galaxy bias at low luminosities because the color cuts applied to select these LRGs will remove blue galaxies that are in the main galaxy sample (see the color distribution plotted in Fig. 3), matching the findings of Zehavi et al. (2005a). This color dependence is not included in our bias model, so we simply exclude the lowest LRG luminosity bin from our final combined sample, removing LRGs with $M_{0.1},>-21.8$. We also exclude all high-luminosity galaxies $M_{0.1_{r}}<-23.0$ (whether main galaxy or LRG), where an expected high bias would have a strong effect on the power spectrum.

We calculate the relative bias of galaxies using the $K$-corrected absolute magnitudes, assuming a flat $\Lambda$ cosmology with $\Omega_{M}=$ 0.3 . The large-scale relative amplitudes of the power spectra of our galaxy subsamples are only weakly dependent on the model chosen to convert from redshift to comoving distance. Consequently, even though we may assume a different cosmology when analyzing the full galaxy sample, we choose to still use the bias as a function of $K$-corrected absolute magnitudes calculated assuming a flat $\Lambda$ cosmology with $\Omega_{M}=0.3$. We do not change the bias model as a function of cosmology.

\section{MEASURING THE POWER SPECTRUM}

The SDSS galaxy power spectrum has been calculated using the Fourier-based method of Percival et al. (2004). Given a model for the average relative bias at each location in the survey, this method extends the method of Feldman et al. (1994) to remove the effect of differential bias from the recovered power spectrum. In addition, this average bias is used to optimally weight the galaxies: more luminous galaxies are stronger tracers of the underlying density field, contain more information about the fluctuations, and therefore should be up-weighted compared with less luminous galaxies.

The Percival et al. (2004) method was used by Cole et al. (2005) to analyze the distribution of galaxies in the 2dFGRS, but there are some differences between our analysis and that of Cole et al. (2005) so we now provide a brief description of the method applied to the SDSS highlighting these differences. The primary change is that, because the sample of galaxies extends to redshift 0.5 , we have dropped the common assumption of using a single cosmological model in order to convert redshifts to comoving radial distances. Instead, we recalculate the power spectrum for different flat $\Lambda$ cosmological models. We sometimes wish to distinguish these cosmologies from those used to create model power spectra. When we do this, we refer to the model used to calculate comoving distances as the "cosmological distance model." Consequently, the data do not compress into a single power spectrum, and there is no single power spectrum resulting from our analysis.

At its heart, the Fourier method provides the simplest way to calculate something approximating the galaxy power spectrum. The galaxies are decomposed onto a grid, the grid is Fourier transformed, and the amplitude of the Fourier modes is measured. One key complication is that weights are applied to each galaxy to optimally reduce the error in the recovered power. Assuming a model for the galaxy bias, the weights applied to a galaxy at location $\boldsymbol{r}$ with expected bias $b^{\prime}$ are those derived in Percival et al. (2004),

$$
w\left(\boldsymbol{r}, b^{\prime}\right)=\frac{\left(b^{\prime}\right)^{2}(\boldsymbol{r}) \bar{P}(k)}{1+\int d b\langle n(\boldsymbol{r}, b)\rangle b^{2} \bar{P}(k)},
$$

where $\bar{P}(k)$ is an estimate of the (unbiased) power spectrum and $\langle n(\boldsymbol{r}, b)\rangle$ is the expected density of galaxies as a function of space and bias. In the analysis presented in this paper we assume $\bar{P}(k)=$ constant $=5000 h^{3} \mathrm{Mpc}^{-3}$, for simplicity. This does not have a strong effect on the accuracy of the recovered power spectrum. The relative weighted contributions from pairs of LRGs, main galaxies, and LRG-main galaxy pairs are plotted as a function of scale in Figure 8. As can be seen, although the LRGs dominate the analysis on all scales, there is a significant contribution to the weighted pair counts from main galaxy-LRG pairs. Had we allowed $\bar{P}(k)$ to be reduced on small scales (as would be optimal), then the higher density regions would contribute more to the weighted pair counts and the main sample galaxies would have dominated the distribution on these smaller scales.

Given a weight $w_{i}$ and expected bias $b_{i}$ for each galaxy, the overdensity field can be written

$$
F(\boldsymbol{r})=\frac{1}{N}\left[\sum_{\text {gal }} \frac{w_{i}}{b_{i}}-\int d b \frac{\langle w(\boldsymbol{r}, b) n(\boldsymbol{r}, b)\rangle}{b}\right],
$$

where $N$ is a normalization constant

$$
N \equiv\left\{\int d^{3} r\left[\int d b\langle w(\boldsymbol{r}, b) n(\boldsymbol{r}, b)\rangle\right]^{2}\right\}^{1 / 2}
$$

and $\langle w(\boldsymbol{r}, b) n(\boldsymbol{r}, b)\rangle$ is the expected weighted distribution of galaxies as a function of bias $b$ and location $\boldsymbol{r}$. We choose to model this field using a random catalog with points selected at the mean galaxy density $\langle n(\boldsymbol{r})\rangle$, using the fits outlined in $\S 2.2$. We use a random catalog containing 10 times as many points as we have galaxies. Because we only need to determine the integral of the 


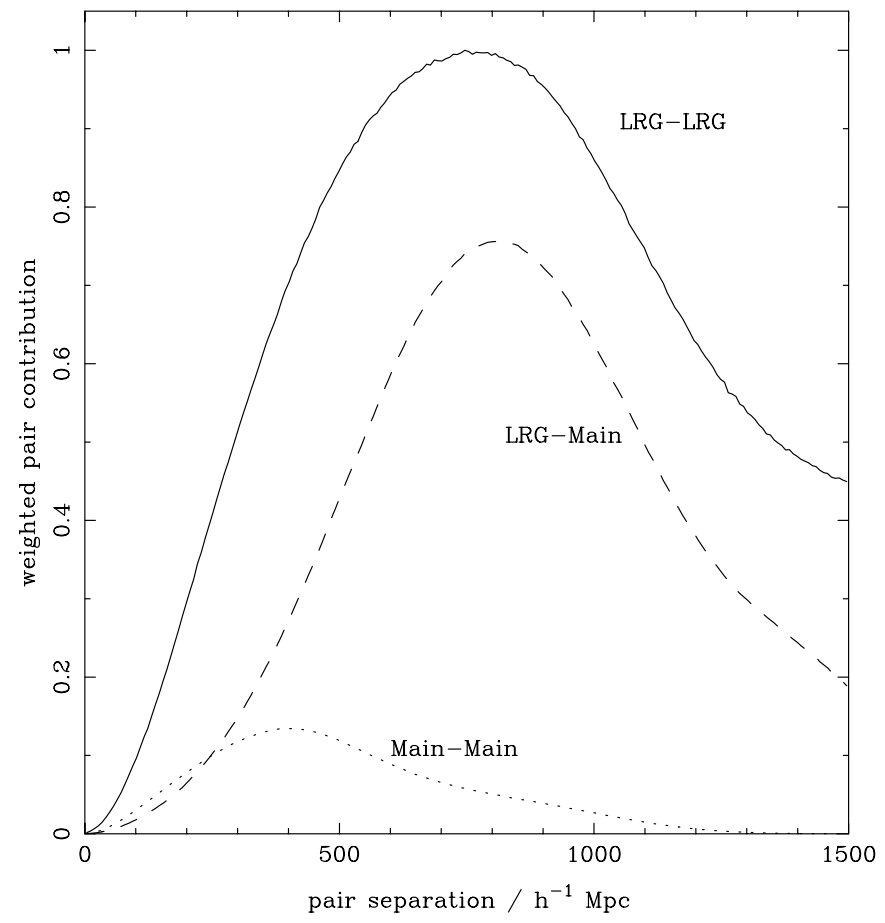

FIG. 8.-Weighted pair counts from LRG-LRG (solid line), main-main (dotted line), and LRG-main (dashed line) pairs. This plot shows how well the correlation function can be measured as a function of scale from these different samples and is directly related to the power spectrum measurement at a particular scale (e.g., Tegmark et al. 2006). The LRG-LRG pairs dominate the measurement on all scales, although there is a significant contribution from pairs formed of an LRG and a main sample galaxy.

average of the weighted and bias-corrected density, we do not need to assign a luminosity to each point in the random catalog and calculate a bias from this. Instead, we simply calculate weights and biases for the random catalog by fitting the average radial values of $w_{i}$ and $w_{i} / b_{i}$ in the galaxy catalog as a function of redshift using cubic splines. The weights in the random catalog are renormalized, compared with the weights applied to the galaxies so that $\int F(\boldsymbol{r}) d r=0$, thereby matching the total weighted number density in galaxy and random catalogs.

The statistic that we use to compare models to the data is the shot-noise-subtracted power spectrum of the overdensity field $F(\boldsymbol{r})$, measured in a series of $k$-space bins. The expectation value of this statistic is

$$
\left\langle|F(\boldsymbol{k})|^{2}-P_{\text {shot }}\right\rangle=\int \frac{d^{3} k^{\prime}}{(2 \pi)^{3}} P\left(\boldsymbol{k}^{\prime}\right)\left|G\left(\boldsymbol{k}-\boldsymbol{k}^{\prime}\right)\right|^{2},
$$

where we average $|F(\boldsymbol{k})|^{2}$ over all $k$-space directions. The shot noise is

$$
P_{\text {shot }}=\sum_{\text {gal }} \frac{w_{i}^{2}}{b_{i}^{2}}+\sum_{\text {ran }}\left\langle\frac{w(\boldsymbol{r}, b)}{b}\right\rangle^{2},
$$

and the window function $|G(\boldsymbol{k})|^{2}$ is the normalized power in a Fourier transform of

$$
G(\boldsymbol{r})=\int d b\langle n(\boldsymbol{r}, b) w(\boldsymbol{r}, b)\rangle .
$$

The spherically averaged window functions from the LRGs, the main galaxies, and the combination of main galaxies and

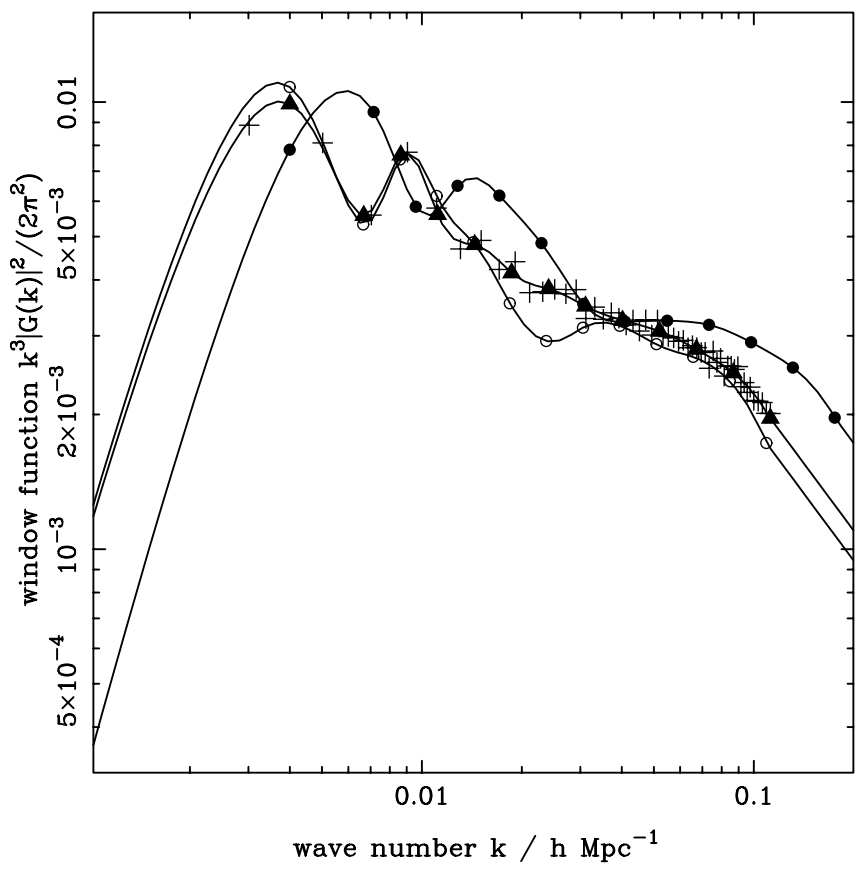

FIG. 9.-Cubic spline fits to the spherically averaged window functions in Fourier space (solid lines) with nodes given by open circles for the SDSS DR5 LRG subsample, filled circles for the main galaxy subsample, and filled triangles for the combined LRG and main galaxy sample. For clarity, we only plot the measured window function power for the combined sample (plus signs). In order to highlight small-scale structure caused by the inhomogeneous nature of the survey, we multiply $|G(k)|^{2}$ by $k^{3}$.

LRGs from the SDSS DR5 sample are compared in Figure 9. The large volume probed by the LRGs means that the $k$-space window is small compared with that calculated for the main galaxies. The window function from the combined LRG and main galaxy sample is close to that of the LRGs, although the main galaxies do provide more pairs of galaxies at intermediate scales, smoothing the structure within the window function. Spline fits to the window functions are used to numerically determine the effect of the window on a model power spectrum. For a smooth power spectrum, the features in the window function are relatively unimportant compared with the overall shape. The correlations induced by the window for the combined main galaxy and LRG sample on the binned power spectrum are plotted in the bottom panel of Figure 10.

The recovered power spectrum values are assumed to be distributed as a multivariate Gaussian, and we estimate the covariance matrix of this Gaussian distribution using lognormal catalogs (Coles \& Jones 1991). For each of 31 flat cosmological distance models with $0.1 \leq \Omega_{M} \leq 0.4$ and $\Delta \Omega_{M}=0.01$, we have created 2000 lognormal catalogs (using the method described in Cole et al. 2005). The distribution of galaxies in these catalogs was calculated using the appropriate cosmological distance model, while the power spectrum was calculated using a linear CDM model (see $\S 5$ ) with parameters chosen to approximately match the amplitude and shape of the recovered power for $0.01 h \mathrm{Mpc}^{-1}<$ $k<0.15 h \mathrm{Mpc}^{-1}$. As seen in $\S 6$, this means that the model power is lower than the power calculated from the data on scales $k<0.02 h \mathrm{Mpc}^{-1}$, so the errors calculated from the lognormal catalogs are probably slightly overestimated on these scales. The diagonal elements of the covariance matrix calculated in this way are based on the effects of cosmic variance and shot noise. The off-diagonal covariance matrix elements include both the effect of the $P(k)$ window functions (Fig. 10, bottom panel) and the 


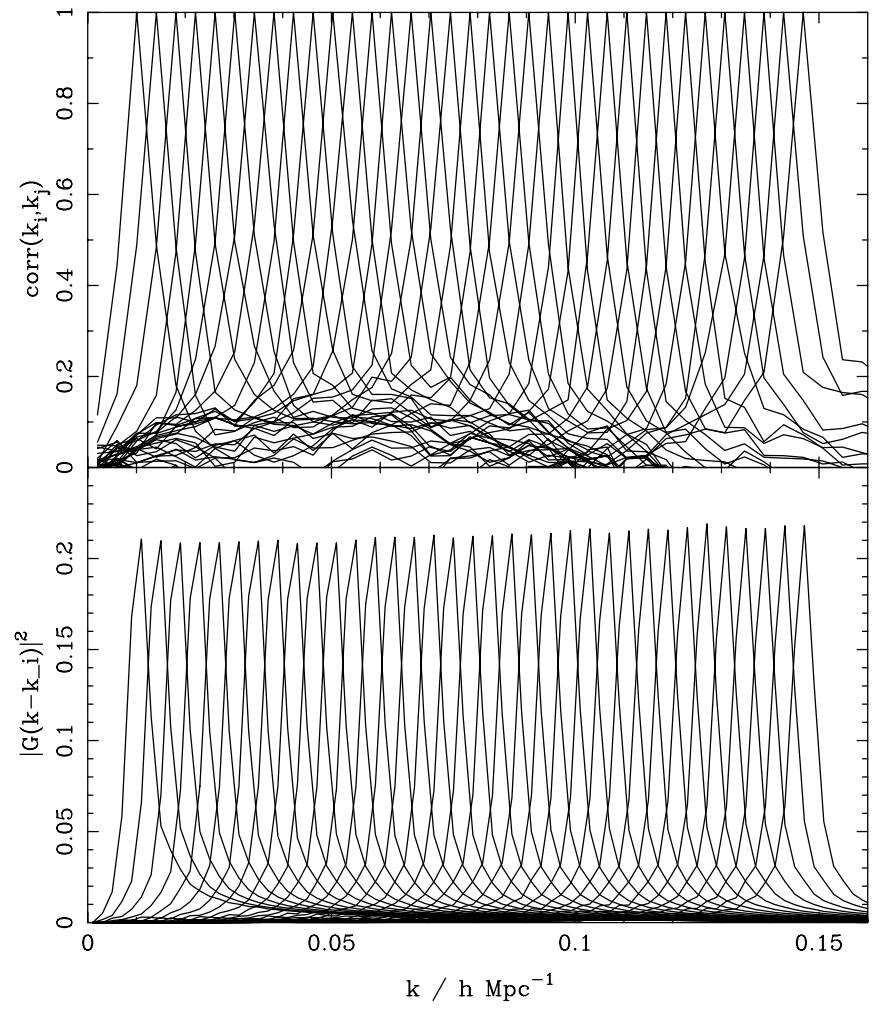

FIG. 10.-Top: Correlations between data values calculated from $2000 \log$ normal catalogs, assuming a flat $\Lambda$ cosmology with $\Omega_{M}=0.24$ for the cosmological distance model. Denoting the covariance between two power spectrum values $P\left(k_{i}\right)$ and $P\left(k_{j}\right)$ as $\operatorname{Cov}\left(k_{i}, k_{j}\right)=\left\langle P\left(k_{i}\right) P\left(k_{j}\right)\right\rangle$, we then plot the correlation between the two measurements given by $\operatorname{Cov}\left(k_{i}, k_{j}\right) /\left[\operatorname{Cov}\left(k_{i}, k_{i}\right) \operatorname{Cov}\left(k_{i}, k_{j}\right)\right]^{1 / 2}$. For presentation, we have calculated the correlations plotted after matching the power spectra amplitudes recovered from the lognormal catalogs. This removes any normalization error and only shows correlations induced by the window function. The covariance matrices used to calculate likelihoods were calculated from the raw power spectra calculated from the lognormal catalogs and therefore include the error in the overall normalization. For $0.01 h \mathrm{Mpc}^{-1}<k<$ $0.15 \mathrm{~h} \mathrm{Mpc}^{-1}$, we see that the correlation between data points is $<0.33$ for $\left|k_{i}-k_{j}\right|>0.01 h \mathrm{Mpc}^{-1}$. Bottom: Normalized window function for each of our binned power spectrum values with $0.01 h \mathrm{Mpc}^{-1}<k<0.15 h \mathrm{Mpc}^{-1}$. Each line shows the relative contribution from the underlying power spectrum as a function of $k$ to the measured data values plotted in Fig. 12. The normalization is such that the area under each line is unity.

mode coupling induced by nonlinear evolution, to the extent that the latter is adequately described by the lognormal approximation. The correlations induced by these effects are shown in the top panel of Figure 10.

The noise in a single covariance matrix element would not normally be noticed when calculating parameter constraints, as it would affect all models in the same way. However, when we use different covariance matrices to test different cosmological models, these errors can become important. Interestingly, if the power spectrum values have a Gaussian distribution, then our estimates of the elements of the covariance matrix will be drawn from a Wishart distribution, the same distribution formed by temperature and polarization CMB power spectra in an all-sky survey (Percival \& Brown 2006). We minimize the effect of this noise by smoothing each element in the set of covariance matrices using a separate four-node cubic spline with nodes at $\Omega_{M}=0.1$, $0.2,0.3$, and 0.4. Three examples of this fit are plotted in Figure 11, for the diagonal elements of the covariance matrix at $k=$ $0.05,0.1$, and $0.15 h \mathrm{Mpc}^{-1}$. There is a clear general trend that the expected error in the power increases with the matter density. This is caused by the changing comoving distance-redshift re-

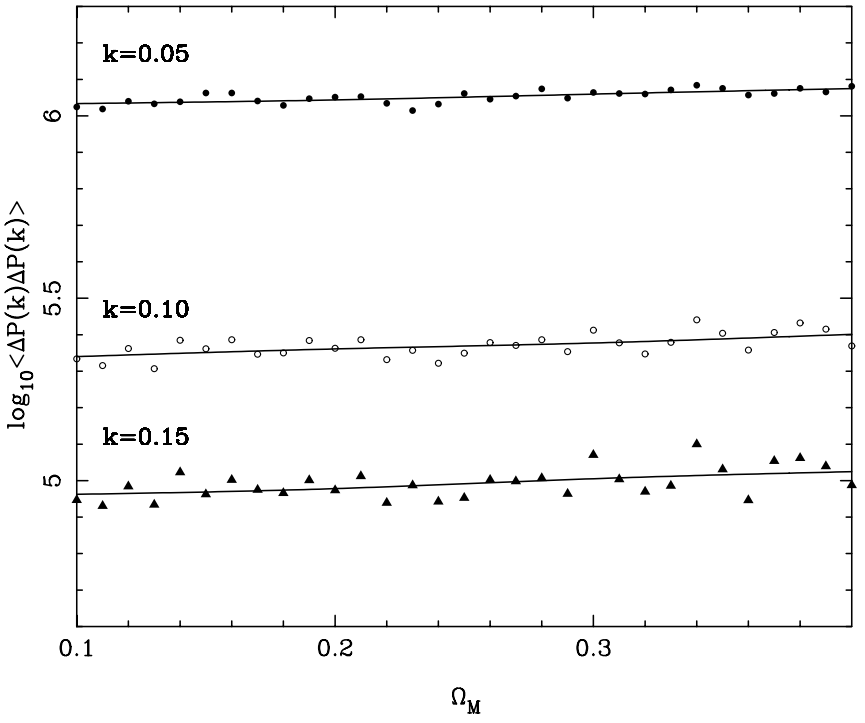

FIG. 11.-Amplitudes of three of the diagonal covariance matrix elements, estimated for flat $\Lambda$ cosmological models with different $\Omega_{M}$, from 2000 lognormal catalogs. These are plotted as a function of $\Omega_{M}$ with $\Delta \Omega_{M}=0.01$. The noise in the individual calculations is clear; this scatter has been minimized by fitting the data with a smooth cubic spline fit shown by the solid lines. We do not plot the error on the covariance matrix (the error in the error) for clarity.

lation, which means that a smaller volume is predicted for the survey assuming $\Omega_{M}=0.4$, rather than $\Omega_{M}=0.1$.

The Fourier transforms used in this paper were performed on $512^{3}$ grids with a varying box size, where we only consider modes that lie between $\frac{1}{4}$ and $\frac{1}{2}$ of the Nyquist frequency for each box. The smoothing effect of the galaxy assignment is corrected as described in Cole et al. (2005). We have compared with both larger $1024^{3}$ and smaller $256^{3}$ Fourier transforms and find no evidence for systematics induced by the practicalities of the Fourier transforms.

Assuming a flat cosmological distance model with $\Omega_{M}=0.24$, matched to the parameters recovered from the $3 \mathrm{yr} W M A P \mathrm{CMB}$ data, the recovered SDSS power spectrum is plotted in Figure 12. Because of the bias model correction, the normalization of this power spectrum is matched to that of $L_{*}$ galaxies, where $M_{*}=$ -20.44 (Blanton et al. 2003b). The precision with which this power spectrum is measured, particularly on large scales, is impressive. In Figure 12 we also plot the linear matter power spectrum predicted from the best-fit $W M A P$ parameters, normalized to match the data on scales $0.01 h \mathrm{Mpc}^{-1}<k<0.06 h \mathrm{Mpc}^{-1}$. This shows good agreement in the shape of the power spectrum on these scales but deviates on scales $k \gtrsim 0.06 h \mathrm{Mpc}^{-1}$. Significant nonlinear corrections to the matter power spectrum are only expected for $k \gtrsim 0.15 h \mathrm{Mpc}^{-1}$ (Smith et al. 2003), and large-scale redshift-space distortions are commonly treated as being scale independent on these large scales. Consequently, it has been common practice to assume that the shape of the power spectrum recovered from galaxy surveys matches the linear matter power spectrum shape on scales $k \lesssim 0.15 h \mathrm{Mpc}^{-1}$ (Percival et al. 2001; Tegmark et al. 2004). However, the SDSS power spectrum has greater power on small scales than the power spectrum predicted by the 3 yr WMAP data.

In Figure 13 we compare the recovered power spectrum with previous power spectra calculated from the SDSS DR3 main galaxy sample (Tegmark et al. 2004) and 2dFGRS (Cole et al. 2005). The error bars on the DR5 data points are much smaller than those on the DR3 data points in part because of the greater sky coverage of DR5 and in (greater) part because of the inclusion 


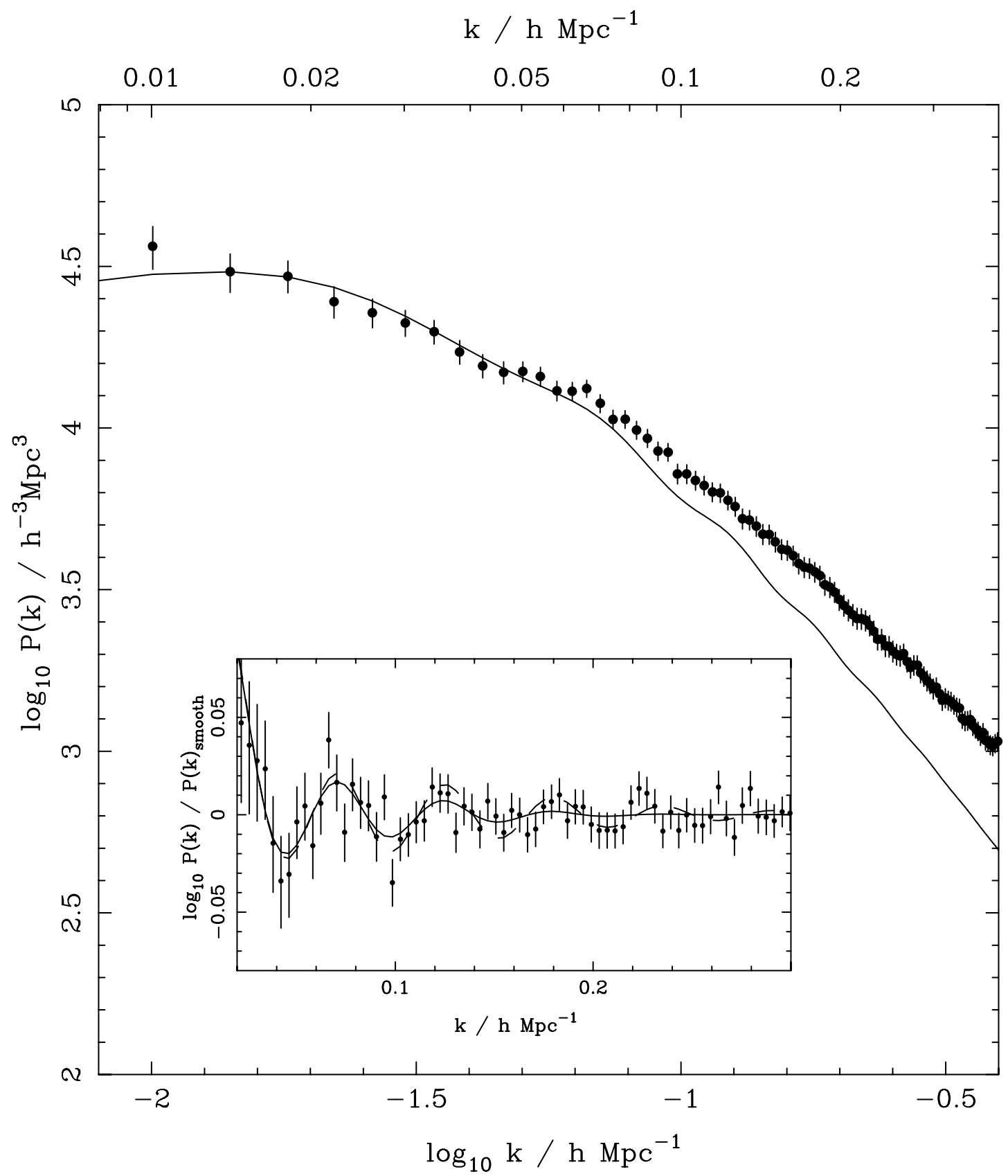

FIG. 12.-Redshift-space power spectrum recovered from the combined SDSS main galaxy and LRG sample, optimally weighted for both density changes and luminosity-dependent bias ( filled circles with $1 \sigma$ errors). A flat $\Lambda$ cosmological distance model was assumed with $\Omega_{M}=0.24$. Error bars are derived from the diagonal elements of the covariance matrix calculated from 2000 lognormal catalogs created for this cosmological distance model, but with a power spectrum amplitude and shape matched to that observed (see text for details). The data are correlated, and the width of the correlations is presented in Fig. 10 (the correlation between data points drops to $<0.33$ for $\Delta k>0.01 \mathrm{~h} \mathrm{Mpc}^{-1}$ ). The correlations are smaller than the oscillatory features observed in the recovered power spectrum. For comparison we plot the model power spectrum (solid line) calculated using the fitting formulae of Eisenstein \& $\mathrm{Hu}$ (1998) and Eisenstein et al. (2006) for the best-fit parameters calculated by fitting the WMAP 3 yr temperature and polarization data, $h=0.73, \Omega_{M}=0.24, n_{s}=0.96$, and $\Omega_{b} / \Omega_{M}=0.174$ (Spergel et al. 2006). The model power spectrum has been convolved with the appropriate window function to match the measured data, and the normalization has been matched to that of the large-scale $\left(0.01 h \mathrm{Mpc}^{-1}<k<\right.$ $0.06 h \mathrm{Mpc}^{-1}$ ) data. The deviation from this low- $\Omega_{M}$ linear power spectrum is clearly visible at $k \gtrsim 0.06 h \mathrm{Mpc}^{-1}$ and is discussed further in $\S 6$. The filled circles with $1 \sigma$ errors in the inset show the power spectrum ratioed to a smooth model (calculated using a cubic spline fit as described in Percival et al. 2007) compared to the baryon oscillations in the (WMAP 3 yr parameter) model (solid line), which shows good agreement. The calculation of the matter density from these oscillations will be considered in a separate paper (Percival et al. 2007). The dashed line shows the same model without the correction for the damping effect of small-scale structure growth of Eisenstein et al. (2006). It is worth noting that this model is not a fit to the data, but a prediction from the CMB experiment.

of LRGs in the sample. It is also worth noting that the procedure used by Tegmark et al. (2004) constructs $P(k)$ estimates that are nearly independent, while our estimates are correlated as shown in Figure 10. After corrections for the window functions and differences in the overall normalization, we see that the large-scale $\left(k \lesssim 0.06 h \mathrm{Mpc}^{-1}\right)$ shape of the power spectrum recovered from the SDSS is well matched to that recovered from the complete 2dFGRS. On smaller scales ( $k \gtrsim 0.06 h \mathrm{Mpc}^{-1}$ ), our SDSS power spectrum of the combined main galaxy and LRG sample reveals more power than that recovered from the 2dFGRS, while the previous power spectrum recovered from the SDSS DR3 main galaxy sample lies between the two. These differences are discussed 


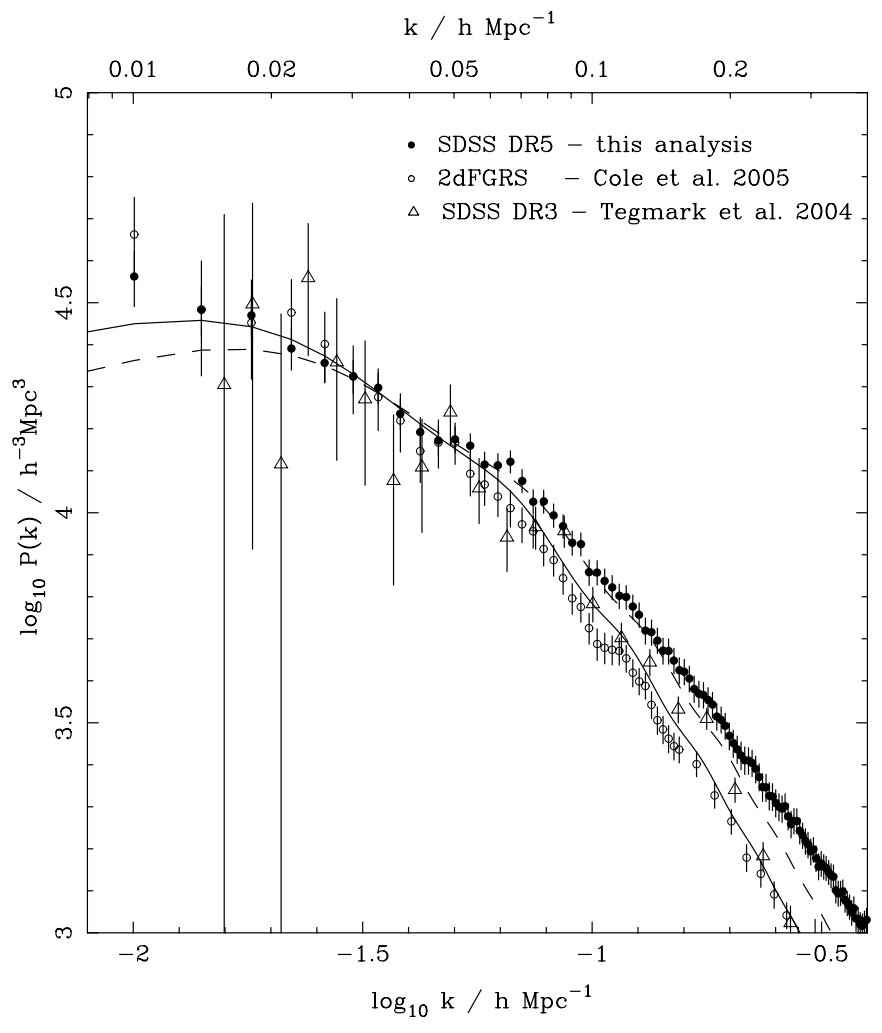

FIG. 13.- Recovered SDSS DR5 power spectrum plotted in Fig. 12 compared with the previous SDSS real-space main galaxy power spectrum calculated for the DR3 sample (from Table 3 of Tegmark et al. 2004) and the 2dFGRS redshift-space galaxy power spectrum of Cole et al. (2005). The data were corrected for the effects of the different window functions by calculating the multiplicative effect on a theoretical model with approximately the correct power spectrum shape. The normalization of the previous data has been matched to that of the new power spectrum on large scales $k<0.06 h \mathrm{Mpc}^{-1}$. The shapes of the power spectra agree well on these scales but predict different power spectrum amplitudes on smaller scales. The solid and dashed lines show two linear CDM model power spectra, plotted as in Fig. 16.

further in $\S$ 9. The power spectrum analysis of the SDSS DR4 LRGs by Huetsi (2006) also showed a consistent power spectrum shape, although the current analysis supersedes this in a number of ways. We analyze a larger sample of galaxies and include an average bias model to correct for the fact that we are analyzing a mixed sample of galaxies. We have also carefully constructed the angular mask of the survey and have provided a number of tests of the analysis method. Consequently, we do not directly compare with the power spectrum derived in this work. In the next section we analyze the shape of the recovered SDSS DR5 power spectrum in detail, looking at the constraints it provides on the matter density.

The 31 sets of power spectra, window functions, and covariance matrices for the combined main-LRG sample, each computed using a different $\Omega_{M}$ for cosmological distance calculations, can be obtained online, ${ }^{18}$ or on request from W. J. P. To use these data to compare the relative likelihood of two model power spectra, one should choose for each model the data set with the closest $\Omega_{M}$ value, convolve the model $P(k)$ with the window functions provided, and calculate the likelihood for each model from the tabulated $P\left(k_{i}\right)$ as a multivariate Gaussian using the corresponding covariance matrix.

\section{MODELING THE GALAXY POWER SPECTRUM}

We calculate linear CDM model power spectra using the fitting formulae of Eisenstein \& Hu (1998) including a correction

\footnotetext{
18 See http://www.dsg.port.ac.uk/ percivalw/.
}

for the damping of the baryon oscillations due to low-redshift small-scale structure (Eisenstein et al. 2006). The damped power spectrum $P_{\text {damped }}(k)$ is assumed to be given by

$$
P_{\text {damped }}(k)=g P_{\text {lin }}(k)+(1-g) P(k)_{\text {smooth }},
$$

where $P_{\text {lin }}$ is the standard linear power spectrum and $P(k)_{\text {smooth }}$ is the same power spectrum without the baryon acoustic oscillations. The parameter $g=\exp \left(-k^{2} \sigma^{2} / 2\right)$ is a Gaussian damping term, and we assume $\sigma=10 h^{-1} \mathrm{Mpc}$ for the spherically averaged redshift-space power spectrum that we measure (Eisenstein et al. 2006).

There are three effects that distort the observed galaxy power spectrum from the linear matter power spectrum. These are nonlinear structure growth, redshift-space distortions, and galaxy bias. Note that we take nonlinear structure growth to correspond to the overall behavior of the matter in the universe, rather than the effect of the collapse of small-scale structures on the galaxy power spectrum, which may be different, depending on how galaxies trace the mass. In this paper we are only concerned with scales where the matter in the universe is still expected to be predominantly in the linear regime $k \lesssim 0.15 h \mathrm{Mpc}^{-1}$ (Smith et al. 2003), so the effect of nonlinear structure growth is small. For the best-fit 3 yr WMAP parameters (Spergel et al. 2006), the Smith et al. (2003) fitting formulae predict an increase in power at $k=$ $0.15 h \mathrm{Mpc}^{-1}$ due to nonlinear effects of $8 \%$. Increasing the value of $\sigma_{8}$ from 0.77 to 0.9 predicts a $10 \%$ increase due to nonlinear effects at $k=0.15 h \mathrm{Mpc}^{-1}$. In contrast, if the linear power spectrum predicted by the $W M A P$ experiment is normalized to the data on scales $0.01 h \mathrm{Mpc}^{-1}<k<0.06 h \mathrm{Mpc}^{-1}$, then the measured SDSS power spectrum is $40 \%$ greater than this model at $k=0.15 h \mathrm{Mpc}^{-1}$.

While redshift-space distortions only depend on the distribution of mass (the galaxies effectively act as test particles within the gravitational potential), the strength of galaxy bias is predicted to depend on galaxy properties. In particular, there is some theoretical work that suggests that we might expect a scale-dependent galaxy bias, even on scales $k \lesssim 0.15 h \mathrm{Mpc}^{-1}$. Seljak (2001) suggests that within the halo model (Seljak 2000; Peacock \& Smith 2000; Cooray \& Sheth 2002), there are two effects that may cause a scale-dependent bias for pairs of galaxies in different halos, which is particularly strong for LRGs (where the galaxies only occupy the most massive halos): if there is an additional Poisson selection of halos to be populated, then there may be a Poisson term in the resulting power spectrum in addition to the standard shot-noise term due to the finite number of galaxies (that is subtracted in the Feldman et al. [1994] method). Such a Poisson term would show up on large scales where the power spectrum has a lower amplitude. In addition, for the most massive halos, the bias is a strong function of the halo mass. Small changes in the average mass of the halos occupied by the galaxies as a function of the scale probed could lead to a scale-dependent galaxy bias.

Using halo model calculations, Cole et al. (2005) introduced a simple two-parameter model (hereafter the $Q$-model) to correct for the effects of galaxy bias and redshift-space distortions, with

$$
P_{\text {gal }}=\frac{1+Q k^{2}}{1+A k} P_{\text {lin }},
$$

where $A=1.4$ and $Q=4.0$ was suggested for the redshift-space $2 \mathrm{dFGRS}$ power spectrum by fitting halo model simulations. For the real-space power spectra, halo model simulations instead predicted that $A=1.7$ and $Q=9.6$. On large scales, the different values of $A$ mean that the redshift-space distortions increase the 
power spectrum slope (so the ratio of small-scale to large-scale power is higher). On small scales the converse is true, and the redshift-space distortion decrease the amplitude of the smallscale power. Between $0.01 h \mathrm{Mpc}^{-1}<k<0.15 h \mathrm{Mpc}^{-1}$, if the model power spectra are normalized on large scales, then this model predicts a $7 \%$ lower redshift-space power spectrum at $k=0.15 h \mathrm{Mpc}^{-1}$ compared with the real-space power spectrum. This is very similar to the offset between linear and nonlinear power discussed above using the Smith et al. (2003) fitting formulae. We see that the real-space power spectrum for the $2 \mathrm{dF}$ galaxies predicted by the halo model tracks the nonlinear increase in power, while the redshift-space distortions effectively cancel the nonlinear increase in power leading to a redshift-space galaxy power spectrum shape that more closely matches that of the linear matter power spectrum.

The two-parameter $Q$-model is used by Tegmark et al. (2006) in their analysis of the SDSS LRG power spectrum; here $A=1.7$ was fixed (corrections for redshift-space effects are included in the method), but $Q$ was allowed to vary to fit the data, with a bestfit value of $Q \simeq 26$ determined from the power spectrum fits in this work. The different values of $Q$ for the SDSS LRGs and $2 \mathrm{dFGRS}$ galaxies reflect a change in shape in the power spectrum, with the LRGs being more biased on small scales relative to large scales. Obviously, in addition to this change in shape, the SDSS LRGs are more biased at any scale than the 2dFGRS galaxies. With the values of $A$ and $Q$ appropriate for the LRGs, the $Q$-model correction to the linear power spectrum varies between 0.98 and 1.01 over $0.01 h \mathrm{Mpc}^{-1}<k<0.06 h \mathrm{Mpc}^{-1}$ and increases on smaller scales to 1.31 at $k=0.15 h \mathrm{Mpc}^{-1}$. It is worth noting that, while the $Q$-model was not designed for such highly biased populations, the modeling presented in Tegmark et al. (2006) suggests that it might still fit the clustering of these luminous galaxies.

Rather than assume such a prescription when fitting models to the data in this paper, we take a step back and instead consider the observational evidence that such a correction is required. First, we test the hypothesis that the observed large-scale power spectrum shape matches that of a CDM linear matter power spectrum over the scales where the matter is predicted to be approximately in the linear regime. We do this by assuming that the observed galaxy power spectrum does match a linear CDM model and then consider if this results in a contradiction in the cosmological parameters derived fitting to different scales. This hypothesis effectively assumes that the contributions to the power spectrum from nonlinear structure growth and redshift-space distortions cancel, while galaxy bias is scale independent. Any contradiction would show (from a single data set) that this cannot be the case. Second, the parameter values $Q \simeq 26$ for the SDSS LRGs and $Q \simeq 4$ for the $2 \mathrm{dFGRS}$ galaxies in the $Q$-model suggest that we should expect a strong change in the shape of the galaxy power spectrum as a function of galaxy properties. We split the large SDSS DR5 sample into subsamples to look for such effects on large scales $0.01 h \mathrm{Mpc}^{-1}<k<0.15 h \mathrm{Mpc}^{-1}$ in $\S \S 7$ and 8 .

\section{6. $\Omega_{M}$ FROM THE POWER SPECTRUM SHAPE}

In this section we fit CDM models to the SDSS DR5 power spectrum data assuming that the observed power spectrum matches the shape of a linear CDM model. We have calculated a grid of model power spectra as described in $\S 5$, allowing the matter density $\Omega_{M}$ and baryon fraction $\Omega_{b} / \Omega_{M}$ to vary, assuming a scalar spectral index of $n_{s}=0.96$ and a Hubble parameter $h=0.73$. Varying $n_{s}$ causes a small change in the recovered value of the matter density: following Cole et al. (2005), the effect can be approximated by $\left(\Omega_{M} h\right)_{\text {true }}=\left(\Omega_{M} h\right)_{\text {apparent }}+0.3\left(1-n_{s}\right)$. Similarly, the effect of a nonzero neutrino fraction would change the recovered

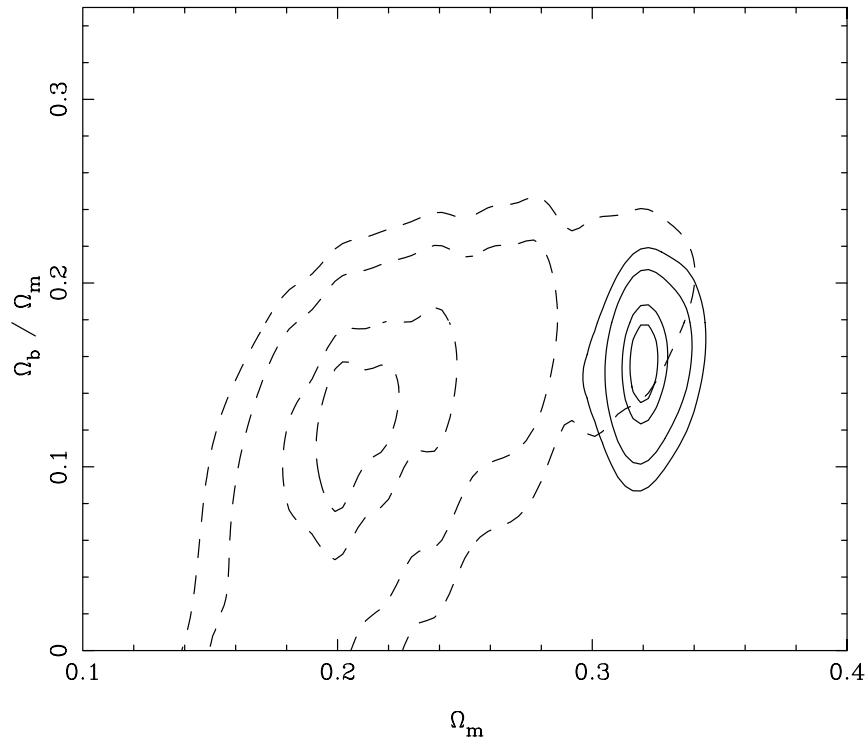

FIG. 14. - Likelihood contours calculated from fitting the power spectrum shape with CDM models. Here $n_{s}=0.96$ and $h=0.73$ are assumed and we vary $\Omega_{M}$ and the baryon fraction. For each value of $\Omega_{M}$ tested, we recalculate the power spectrum, the window function, and the expected error on the power so the cosmological distance model matches the power spectrum model. Dashed contours were fitted for $0.01 h \mathrm{Mpc}^{-1}<k<0.06 h \mathrm{Mpc}^{-1}$, and solid contours for $0.01 h \mathrm{Mpc}^{-1}<k<0.15 h \mathrm{Mpc}^{-1}$. Contours are plotted for $-2 \ln \mathcal{L}=$ $1.0,2.3,6.0$, and 9.2 , corresponding to one-parameter confidence of $68 \%$ and two-parameter confidence of $68 \%, 95 \%$, and $99 \%$ for a Gaussian distribution.

value by approximately $\left(\Omega_{M} h\right)_{\text {true }}=\left(\Omega_{M} h\right)_{\text {apparent }}+1.2\left(\Omega_{\nu} / \Omega_{M}\right)$. For each value of the matter density tested, we have recalculated the window function from the geometry of the sample using the appropriate comoving distance-redshift relation. This window function is used to convolve the model power and is used to correct for the loss of power due to the normalization of the overdensity field: because the total expected number of galaxies is unknown, the normalization of the random catalog was matched to the galaxy catalog so that $\int F(r) d r=0$ (see eq. [3] and the subsequent discussion). The effect of this on the data is to subtract a multiple of the window function, so that $P_{\text {data }}(0)=0$. We therefore subtract a multiple of the window function from the model power spectra (after convolution with the appropriate window) so that $P_{\text {model }}(0)=0$. This is the equivalent of the "integral constraint" correction required for measured correlation functions. The likelihoods of the model power spectra are then calculated assuming that the data form a multivariate Gaussian distribution with the appropriate covariance matrix, calculated as described in $\S 4$.

The resulting likelihood surfaces are plotted in Figure 14. Contours are plotted for fits to two different $k$-ranges, $0.01 h \mathrm{Mpc}^{-1}<$ $k<0.06 h \mathrm{Mpc}^{-1}$ (dashed contours) and $0.01 h \mathrm{Mpc}^{-1}<k<$ $0.15 h \mathrm{Mpc}^{-1}$ (solid contours). As can be seen, the choice of scales fitted makes a strong difference to the recovered best-fit parameters. On scales $0.01 h \mathrm{Mpc}^{-1}<k<0.06 h \mathrm{Mpc}^{-1}$, the shape matches that of a low matter density cosmology with $\Omega_{M}=$ $0.22 \pm 0.04$. Extending the fit to smaller scales, the data prefer a higher matter density $\Omega_{M}=0.32 \pm 0.01$. The fits are discrepant at the 2-3 $\sigma$ level. The corresponding marginalized baryon fractions are $\Omega_{b} / \Omega_{M}=0.13 \pm 0.05$ and $\Omega_{b} / \Omega_{M}=0.16 \pm 0.03$. On scales $0.01 h \mathrm{Mpc}^{-1}<k<0.06 h \mathrm{Mpc}^{-1}$, the small-scale damping does not strongly affect the recovered power and we recover the same parameter constraints if we do not make the smallscale damping correction to the model baryon acoustic oscillations. Fitting to $0.01 h \mathrm{Mpc}^{-1}<k<0.15 h \mathrm{Mpc}^{-1}$ without 


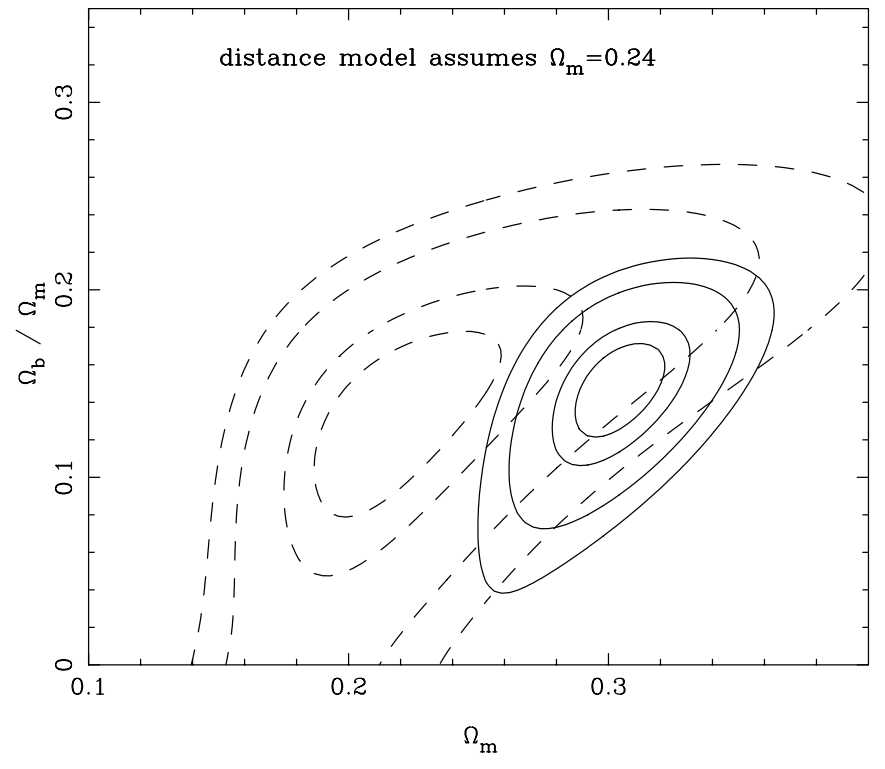

Fig. 15.- Same as Fig. 14, but now only considering a single power spectrum, calculated assuming a flat $\Omega_{M}=0.24$ cosmology to determine comoving galaxy distances from the measured galaxy redshifts.

the damping term reduces the baryon fraction constraint slightly to $\Omega_{b} / \Omega_{M}=0.15 \pm 0.02$.

The results from fitting the power spectrum shape are only weakly dependent on the model assumed to calculate comoving distances from galaxy redshifts. We have considered fitting general CDM models to the single power spectrum calculated assuming a fixed cosmological distance model with $\Omega_{M}=0.24$ (as plotted in Fig. 12), and likelihood contours are presented in Figure 15, as for Figure 14. The general form of the contours remains consistent, although the locations of the likelihood maxima do change: for the fit to $0.01 h \mathrm{Mpc}^{-1}<k<0.15 h \mathrm{Mpc}^{-1}$ we recover $\Omega_{M}=0.31 \pm 0.02$.

The best-fit models are compared with the data in Figure 16, which clearly shows why the discrepancy arises. There is too much power on scales $0.01 h \mathrm{Mpc}^{-1}<k<0.06 h \mathrm{Mpc}^{-1}$ for the $\Omega_{M}=0.32$ model at approximately $1 \sigma$. Conversely, for the fit to the large-scale data, there is too much power on smaller scales $k>0.06 h \mathrm{Mpc}^{-1}$.

\section{POWER SPECTRA OF SUBCATALOGS AND ROBUSTNESS TESTS}

In this section we present a number of test power spectra, calculated for variations of our method or for different subsamples of the data. The power spectra were calculated using a flat cosmological model with $\Omega_{M}=0.24$ to convert from redshifts to comoving distance and are compared in Figures 17 and 18. In the left column they are divided by a linear power spectrum calculated with no baryons and with $\Omega_{M}=0.18$, which has approximately the correct shape but no baryonic features, and the right column shows the same model convolved with the appropriate window function for each power spectrum and with matched normalization. For comparison we plot the power spectrum recovered using our standard analysis method in the top row of Figure 17. The tests are described as follows:

1. We have split the catalog into (slightly overlapping) main galaxy and LRG subsamples and have calculated power spectra for these samples using our standard method and our standard luminosity-dependent bias model. Figure 17 shows that the largescale normalizations of the recovered power spectra match well

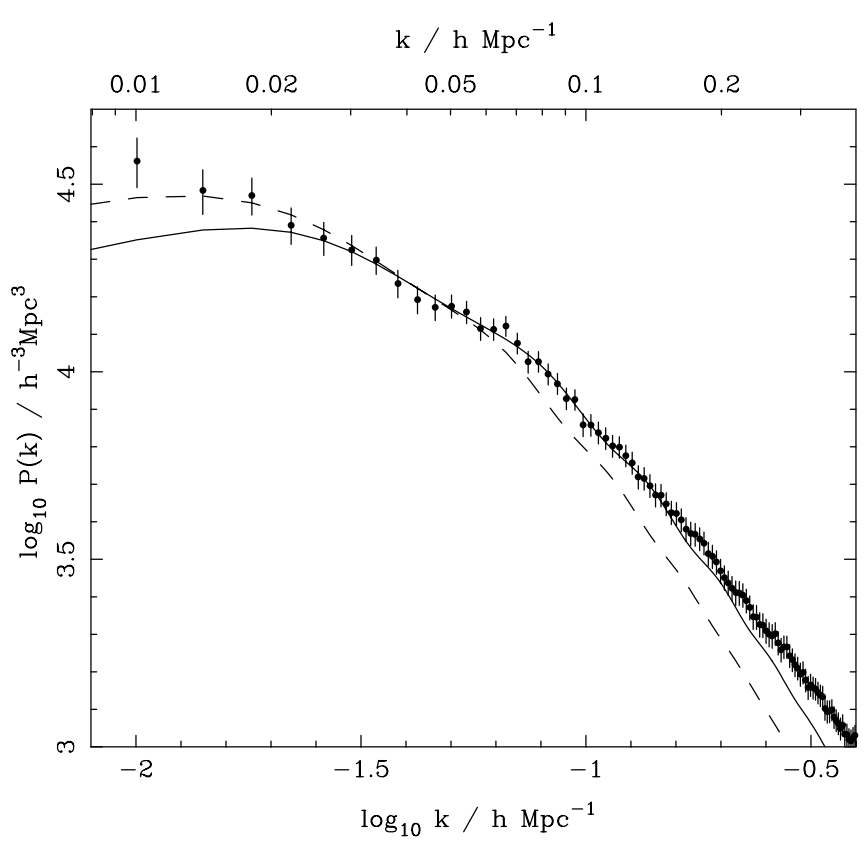

FIG. 16.-Power spectra recovered from the combined SDSS DR5 sample assuming $\Omega_{M}=0.24$ to convert from redshifts to comoving distances ( filled circles with $1 \sigma$ errors) compared with the best-fit CDM models. The dashed line shows the best-fit model over $0.01 h \mathrm{Mpc}^{-1}<k<0.06 h \mathrm{Mpc}^{-1}$, while the solid line shows the best-fit model over $0.01 h \mathrm{Mpc}^{-1}<k<0.15 h \mathrm{Mpc}^{-1}$. The two fits are discrepant at approximately $2-3 \sigma$.

compared with the relative average bias of the two populations. This shows that the bias model used is renormalizing both power spectra to match that of an $L_{*}$ galaxy. There is weak evidence for a small normalization offset, matching the small offset in the measured LRG bias compared with the model assumed (the open circles are slightly above the dashed line in Fig. 6). We test the bias model later and show that small deviations in the bias assumed for the LRGs compared with the main galaxies do not affect the power spectrum calculated from the combined sample (test 5). On small scales the LRG power has a higher amplitude compared with the main galaxy power.

2. We test our angular mask by comparing recovered power spectra from samples of main galaxies selected to a limiting magnitude of $r<17.5$ using the angular and radial selection methods described in $\S 2$, with a similar catalog obtained from the SDSS New York University Value Added Catalogue (NYU-VAGC; Blanton et al. 2005). When analyzing the NYU-VAGC sample, we used their angular mask, which therefore matches the mask derivation previously used for SDSS team analyses (Tegmark et al. 2004, 2006; Eisenstein et al. 2005), although this calculation has obviously been updated to cover the SDSS DR5 region. There are small changes between the NYU-VAGC mask and our mask, due to slightly different selection criteria and regions covered, but in the areas of overlap they match well. We select a "safe" sample of main galaxies with a magnitude limit of $r<17.5$ in order to provide a sample with a single radial distribution and avoid complications from differing faint survey limits. For the NYU-VAGC we use the model provided for the radial distribution of galaxies, which was calculated from fits to the luminosity function as described in Tegmark et al. (2004). For our sample we calculate the radial distribution of galaxies as described in $\S 2.2$. Even though these independent methods vary substantially in design for both the angular and radial selection, as can be seen in Figure 17, there are only very minor differences between the recovered power spectra. 


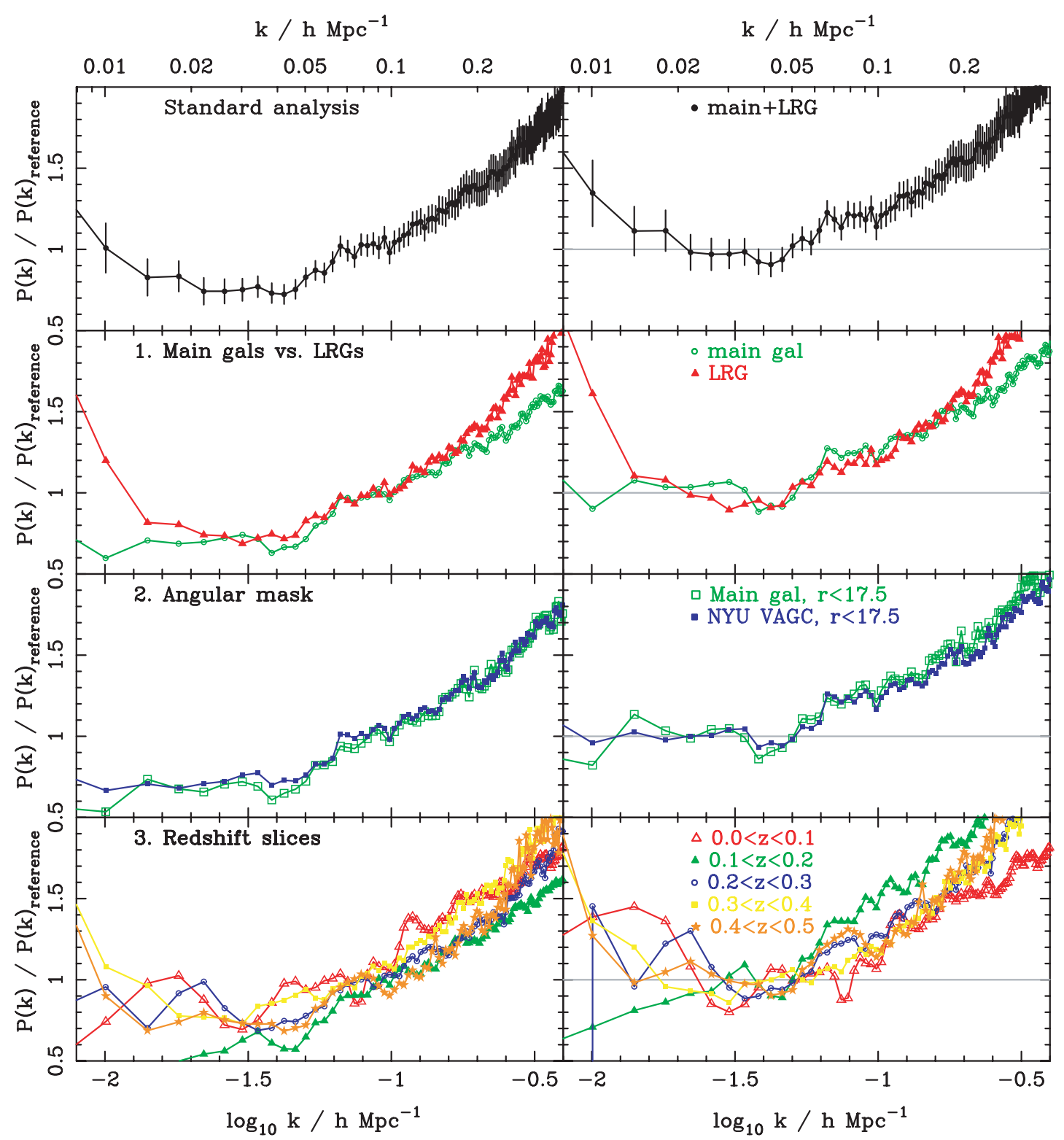

FIG. 17. - Power spectra calculated from the SDSS DR5 sample divided by a model linear power spectrum with $h=0.73, \Omega_{M}=0.18, n_{s}=0.96$, and no baryons (in order not to introduce extra oscillations in the plotted power spectra). In the left column, we simply divide by the raw model power spectrum, with a fixed normalization matched to the final power spectrum from the combined LRG + main galaxy sample. In the right column we convolve the model power spectrum with the window function appropriate to the test being performed and correct for the loss of power due to the normalization of the overdensity field, before calculating the ratio of the power spectra. The amplitude is allowed to vary to match the data on scales $0.01 h \mathrm{Mpc}^{-1}<k<0.06 h \mathrm{Mpc}^{-1}$, so we are only comparing the shapes of the power spectra recovered from the different tests in the right column. The top row shows the ratios calculated for our final power spectrum derived from the combined main galaxy and LRG catalog. The other rows show power spectra calculated either for different subsamples of this catalog or using slightly different techniques, described in $\S 7$.

3. We test the radial distribution assumed for the galaxy population by comparing the power spectra measured in five redshift slices of width $\Delta z=0.1$ through the combined sample of main galaxies and LRGs. The standard luminosity-dependent bias model is assumed and appears to adequately renormalize the power spectra calculated from the different samples: there is no evidence for a significant change in normalization of the power as a function of redshift. However, the magnitude-limited catalogs from which the bias model was derived have redshift increasing with luminosity. The derivation of the model is therefore coupled with any redshift evolution. Consequently, it is perhaps not surprising that the amplitudes of the power spectra recovered in different redshift slices are so similar, although it is still gratifying to see that this is correct. A simple model with constant galaxy clustering as a func- tion of redshift and with redshift-independent luminosity-dependent bias is therefore sufficient to model the currently observed clustering but is not necessarily a unique solution.

4. The main galaxy sample is bimodal in color; we have decomposed this data set into red and blue galaxy subsamples using a simple color cut $M_{0.1_{g}}-M_{0.1_{r}}=0.8$ (see Fig. 3). These subsamples were analyzed in exactly the same way as our final combined sample. In particular, the redshift distribution fitting function of equation (1) was found to still provide an adequate match to the observed redshift distribution. The power spectra for these subsamples were corrected using our bias model, so the change in normalization of the power spectra of the red and blue subsamples demonstrates the additional color-dependent bias term that is not included in our average bias model. As can be seen, there is no 


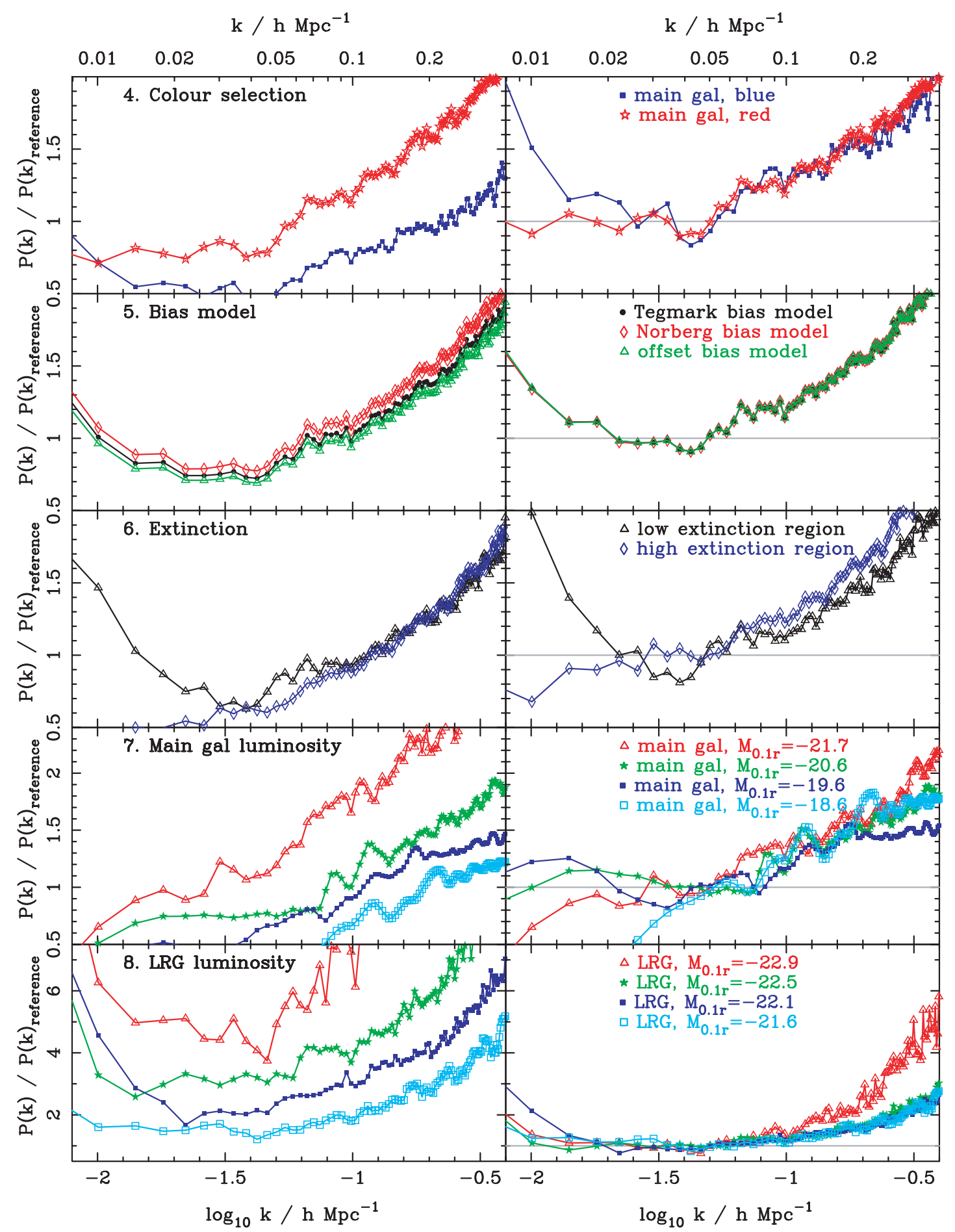

FIG. 18. - Continuation of Fig. 17. To accommodate the range of power spectrum normalizations in tests 7 and 8 , the scale of the $y$-axis was changed in these panels.

obvious change in the shape of the power spectra as a function of color.

5. We also consider a power spectrum calculated using the bias model of Norberg et al. (2001) rather than the bias model of Tegmark et al. (2004). The primary effect is a change of normalization, equivalent to a change in the definition of $b_{*}$. Once this is corrected in the right column of Figure 18, we see no significant change in the recovered power spectra. We also consider an "offset" bias model, where we assume a model bias for the LRGs given by $b / b_{*}=0.85+0.15 L / L_{*}+0.08\left(M_{*}-M_{0.1_{r}}\right)$, but do not change the bias model for the main galaxies. If this model is used to calculate the power in the LRG and main galaxy catalogs separately, the recovered large-scale power spectrum amplitude recovered from the LRGs is reduced, and the LRG and main galaxy power spectra are in better agreement (see test 1 ). For the combined sample (or the LRG or main galaxy samples if analyzed independently), this change does not affect the overall shape of the power spectrum, giving us confidence that any error introduced by joining the two galaxy catalogs is not significant.

6. The angular coverage of the SDSS sample is now sufficiently large that we can split the catalog as a function of Galactic extinction. The median $r$-band extinction in the sample is 0.065 , 


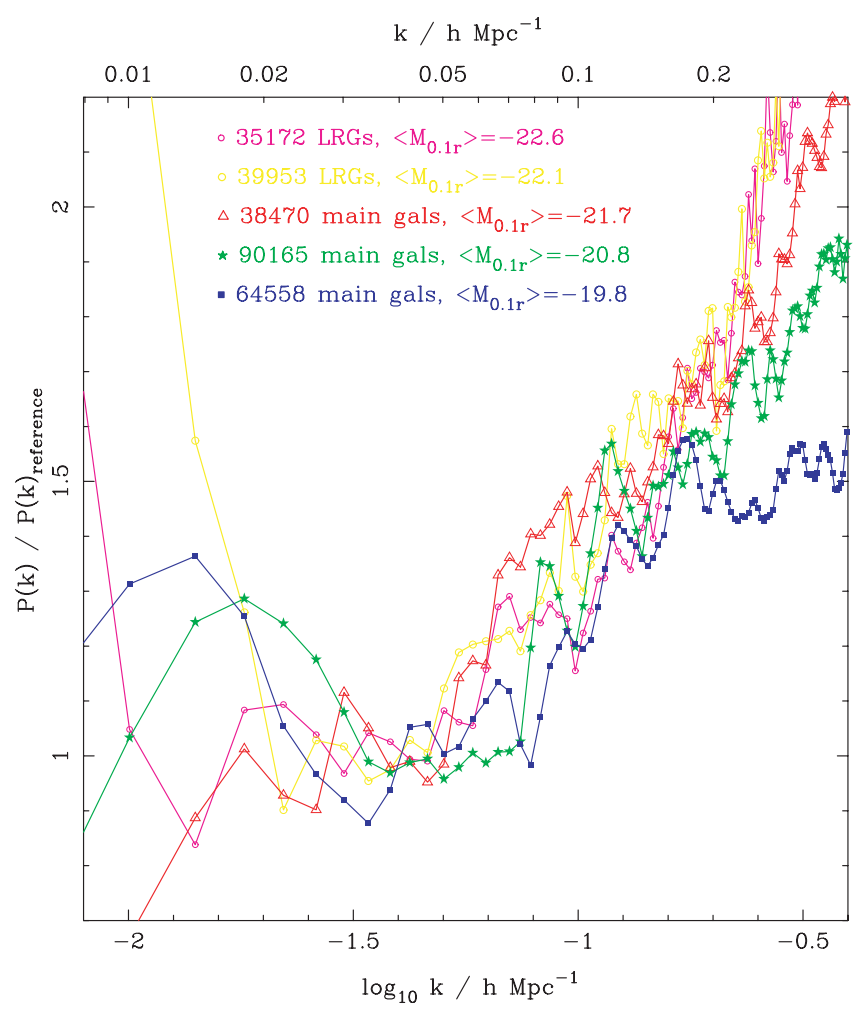

FIG. 19.- Power spectra calculated from LRG and main galaxy subcatalogs divided by a smooth CDM model matched to the subcatalog (as in the right panels of Fig. 17). The amplitude of the CDM model was matched to the data over the narrow $k$-range $0.03 h \mathrm{Mpc}^{-1}<k<0.05 h \mathrm{Mpc}^{-1}$. For the main galaxies, three pseudo-volume-limited catalogs of width 1 mag covering $-22.5<$ $M_{r}<-19.5$ were created and analyzed (as described in $\S 3.1$ ). For the LRGs, the catalog was split at approximately the median luminosity of the sample $M_{0.1_{r}}=-22.3$.

and we split into galaxies with a higher extinction correction and galaxies with a lower extinction correction. The recovered power spectra are not independent, and we have not calculated relative errors for these data. However, the power spectra diverge on scales $k<0.02 h \mathrm{Mpc}^{-1}$ and are also slightly discrepant on scales $k=$ $0.05 h \mathrm{Mpc}^{-1}$, which could indicate a systematic problem with the extinction corrections. A recent test of the number density of SDSS galaxies as a function of the Schlegel et al. (1998) Galactic extinction correction was performed by Yahata et al. (2006). They find that the number density of galaxies increases with increasing extinction for SFD extinction values below $0.1 \mathrm{mag}$ in the $r$ band. The contamination of the far-infrared brightness of the sky by background galaxies, postulated as an explanation of this effect in this work, might also explain the observed difference between these power spectra.

7. The final tests presented in Figure 18 compare power spectra recovered for galaxies of different luminosity. For the main galaxies, we plot four of the nine pseudo-volume-limited catalogs described in $\S 3$. Each catalog is 0.5 mag wide (not $K$-corrected), and no bias model has been included. Consequently, the increase in the normalization of the large-scale clustering as a function of luminosity is clear in the left column.

8. As for test 7, but now comparing four LRG subcatalogs, each of width $0.5 \mathrm{mag}$ (not $K$-corrected). Looking at the left column, the increase in the overall clustering strength with luminosity is clear. In the right column, we see that any change in the shape of the power spectrum as a function of luminosity is at a level significantly below the change in the large-scale normal- ization. The possibility that the power spectrum does change shape on scales $0.01 h \mathrm{Mpc}^{-1}<k<0.15 h \mathrm{Mpc}^{-1}$ as a function of luminosity is considered further in the next section.

\section{OBSERVED CLUSTERING AS A FUNCTION OF LUMINOSITY}

In this section we expand on tests 7 and 8 presented in $\S 7$ in order to consider whether there is any evidence that the power spectrum changes shape as a function of luminosity on scales $0.01 h \mathrm{Mpc}^{-1}<k<0.15 h \mathrm{Mpc}^{-1}$. In order to test this, we have created three pseudo-volume-limited subcatalogs of the main galaxy sample, using the method described in $\S 3.1$, but now each of width $1 \mathrm{mag}$, covering $-22.5<M_{r}<-19.5$ (without $K$-correction). We have also split the LRG sample into two cata$\operatorname{logs}$ at approximately the median magnitude $M_{0.1_{r}}=-22.3$. Power spectra have been calculated for these five subcatalogs and are plotted in Figure 19, divided by a fiducial power spectrum model matched to each power spectrum as in the right column of Figures 17 and 18.

With the amplitude of the data matched on large scales, on small scales $k>0.2 h \mathrm{Mpc}^{-1}$, there is a clear hierarchy with more luminous galaxies showing stronger small-scale clustering. In order to quantify the effect on cosmological parameter estimation in the regime where the matter is still linear $\left(k \lesssim 0.15 h \mathrm{Mpc}^{-1}\right)$, the important question is, on what scale do the power spectra start to deviate from each other? Unfortunately, the data are insufficient to answer this question in a quantitative way. For the samples split from the main galaxy catalog, there is perhaps a slight trend with the least luminous galaxies having less power on scales $0.06 h \mathrm{Mpc}^{-1}<k<0.15 h \mathrm{Mpc}^{-1}$, but this conclusion is fairly speculative.

\section{DISCUSSION}

We have analyzed the clustering of galaxies in the largest sample (in terms of number and volume covered) to date. We have shown that the SDSS main galaxy and LRG samples can be naturally combined because both sets of galaxies, after careful luminosity selection, can be described using the same average large-scale luminosity-dependent bias model. Analyzing the combination of data allows us to include cross-correlation between the two data sets, as well as internal autocorrelations within either the LRG or main galaxy samples.

Because of the speed and simplicity of the Fourier method used, we have been able to consider a number of tests of the recovered power spectrum and have eliminated a number of possible systematics from our analysis. The only unusual result was a possible problem with the Galactic extinctions; this requires further detailed analysis and modeling that is outside the scope of our paper. In addition to considering a larger data set than previously analyzed, our analysis extends previous work by considering the cosmological model to be tested from the start of the analysis, using this model to convert from redshift to comoving distance, as well as comparing with the resulting power spectrum.

In a companion paper we have considered the cosmological constraints from the baryon acoustic oscillations observed in the power spectrum, where we found $\Omega_{M}=0.250_{-0.021}^{+0.042}$ for flat $\Lambda$ cosmological models (Percival et al. 2007) when combined with the observed baryon acoustic oscillation scale in the CMB (Spergel et al. 2006). This paper has instead focused on trying to model the overall shape of the power spectrum, paying particular attention to systematic effects on the resulting matter density constraints. Because of the accuracy with which we can now measure the power spectrum on scales $k<0.05 h \mathrm{Mpc}^{-1}$ 
as a consequence of the large volume covered by the LRG sample, we can test the hypothesis that the shape of the redshift-space galaxy power spectrum matches the matter on scales where the matter clustering is well modeled by linear evolution from the seed perturbations $\left(0.01 h \mathrm{Mpc}^{-1}<k<0.15 h \mathrm{Mpc}^{-1}\right)$. We find a discrepancy between fits to $k<0.06 h \mathrm{Mpc}^{-1}$ and $0.06 h \mathrm{Mpc}^{-1}<$ $k<0.15 h \mathrm{Mpc}^{-1}$, albeit at only moderate significance. The data on scales $0.01 h \mathrm{Mpc}^{-1}<k<0.06 h \mathrm{Mpc}^{-1}$ favor a model with $\Omega_{M}=0.22 \pm 0.04$, while extending the $k$-range to $0.01 h \mathrm{Mpc}^{-1}<k<0.15 h \mathrm{Mpc}^{-1}$ increases the estimated value to $\Omega_{M}=0.32 \pm 0.01$. Statistically, this discrepancy is only at the 2-3 $\sigma$ level. It is worth noting that the excess power on scales $k<0.06 h \mathrm{Mpc}^{-1}$ relative to the $\Omega_{M}=0.32$ model matches that recently found from analyses of the SDSS photometric redshift sample (Padmanabhan et al. 2006; Blake et al. 2007). This suggests that the discrepancy is therefore not limited to our analysis. The upper $k$ limit for the large-scale fit, $k_{\lim }=0.06 h \mathrm{Mpc}^{-1}$, was conservatively chosen based on the observed shape of the power spectrum (Fig. 12). The noise in the data means that there is nothing special in this choice, and we could have split the scales fitted at $0.05 h \mathrm{Mpc}^{-1}<k_{\lim }<0.1 h \mathrm{Mpc}^{-1}$ and obtained similar results.

Interestingly, the change in the recovered matter density with the scales fitted matches the bimodality of previous matter density constraints, with the best-fit model on scales $0.01 h \mathrm{Mpc}^{-1}<$ $k<0.06 h \mathrm{Mpc}^{-1}$ matching that predicted by the WMAP $3 \mathrm{yr}$ data (Spergel et al. 2006), the 2dFGRS (Cole et al. 2005), and the results from the baryon acoustic oscillations observed in the SDSS DR5 sample. In contrast, the fits to $0.01 h \mathrm{Mpc}^{-1}<k<$ $0.15 h \mathrm{Mpc}^{-1}$ match the results from early studies of the SDSS data by Pope et al. (2004) and Tegmark et al. (2004), suggesting that we are recovering approximately the same overall shape on these scales as these early analyses. The new data obviously constrain $\Omega_{M} h$ with greater accuracy so, on the scales previously considered, the significance of the offset between 2dFGRS and SDSS analyses has increased. In addition, the higher value of $\Omega_{M}=0.32 \pm 0.01$ is now more discrepant with the (mean) $W M A P$ constraint $\Omega_{M}=0.234 \pm 0.034$ (from Table 2 of Spergel et al. 2006) and is discrepant with the lower matter density recovered from the positions of the baryon acoustic oscillations in the power spectrum (Percival et al. 2007).

The hypothesis being tested by these model fits is that the contributions to the power spectrum from nonlinear structure growth and redshift-space distortions cancel, galaxy bias is scale independent, and the matter clusters as expected in a simple CDM model with an unbroken power spectrum. The results provide a contradiction to this hypothesis with a significance of 2-3 $\sigma$. We now consider each of the elements in turn to try to understand which assumption is breaking down. First, it is worth noting that the effect of nonlinear structure growth was discussed in $\S 5$ and shown to be too small to cause the observed power spectrum distortion.

Small-scale redshift-space distortions act in the wrong direction for the observed effect, and it would require a significant breakdown of the scale-independent increase in power spectrum amplitude predicted by Kaiser (1987) for linear infall observed at large distances to give a scale-independent increase in the power spectrum amplitude and cause the observed change in shape. $N$-body simulations and the halo model calculations performed for the $2 \mathrm{dFGRS}$ also found that the effect of redshift-space distortions on the shape of the power spectrum is small on the scales of interest (Percival et al. 2001; Cole et al. 2005). The consistency between the higher matter density favored fitting to $0.01 h \mathrm{Mpc}^{-1}<k<0.15 h \mathrm{Mpc}^{-1}$ in our study, previous studies of the real-space power spectrum (Tegmark et al. 2004), and the power spectrum calculated from sets of photometric redshift LRGs also provides evidence against redshift-space distortions producing the observed effects.

An unwelcome possibility is a systematic problem with one of the data sets, or the analysis method, although the only discrepancy revealed in the tests performed in $\S 7$ was between the power spectra recovered using different Galactic extinction correction cuts. However, this potential systematic could also explain differences between 2dFGRS and SDSS power spectra if it predominantly affects the SDSS galaxies; analyzing the galaxy clustering in the low-extinction regions in the SDSS, for which Yahata et al. (2006) show that the number density of galaxies does not behave as expected, produces a slight excess of power on scales $k \simeq$ $0.06 h \mathrm{Mpc}^{-1}$ (see Fig. 18). In addition, it is perhaps also worth mentioning the heretical possibility that there is a problem with the assumption that the matter clusters as expected in a CDM model with an unbroken postinflation power-law spectrum. However, such a problem would affect the 2dFGRS and SDSS galaxies in the same way, so a further explanation would be required for this difference.

Perhaps the most simple explanation for this inconsistency is that the LRGs that dominate our combined sample do not trace the linear matter power spectrum as simply as other galaxies, and there is some theoretical work that supports this assertion. In order to try to obtain evidence for scale-dependent galaxy bias, we have analyzed the shape of the SDSS power spectrum, particularly looking for evidence of an increase in small-scale clustering power that depends on galaxy properties. The luminosity bias correction applied as part of the Fourier method will only correct for the large-scale, scale-independent bias affecting different luminosity galaxies. It is not designed to correct for scaledependent bias. We have analyzed a number of subsets of our final catalog in order to look for scale-dependent bias and find no significant change in shape if we split our sample using the bimodal galaxy color distribution, or if we split in redshift; we recover similar power spectrum shapes, analyzing the data in five redshift slices out to $z=0.5$. There is weak evidence for a change in the shape of the power spectrum for $0.01 h \mathrm{Mpc}^{-1}<$ $k<0.15 h \mathrm{Mpc}^{-1}$ when splitting the galaxies by luminosity, which varies with the average $r$-band luminosity of the galaxy sample analyzed. However, the evidence for this is not conclusive. It is worth noting that we clearly see the effects of color and luminosity on smaller scales $k>0.2 h \mathrm{Mpc}^{-1}$, in line with the results of Cole et al. (2005). If scale-dependent bias also affects large scales, then, in principle, this is testable by measurement of the bispectrum on the same data set (R. Scoccimarro et al. 2007, in preparation): the bispectrum shape and scale dependence respond to bias in a way that differs from, and is therefore not degenerate with, the power spectrum.

A change in power spectrum shape on scales $k<$ $0.15 h \mathrm{Mpc}^{-1}$, as a function of luminosity, would provide a consistent picture when we compare our recovered power spectrum with previous work. On scales $k \lesssim 0.06 h \mathrm{Mpc}^{-1}$, Figure 13 shows that the shape is consistent with the $2 \mathrm{dFGRS}$ power spectrum (Cole et al. 2005). If the amplitudes of the power spectra are matched on these large scales, then on smaller scales there is a progression from the low clustering amplitude of the 2dFGRS galaxies, through the main galaxies of the DR3 SDSS sample analyzed by Tegmark et al. (2004), to the higher clustering strength of the combined main galaxy and LRG sample of this analysis. There is no reason to suggest that this is not a natural progression following the trend observed within the SDSS from galaxies with a low $r$-band luminosity to those with a high $r$-band luminosity. 
In this interpretation, the "excess power" on the largest scales probed by the Padmanabhan et al. (2006) and Blake et al. (2007) analyses of photometric LRGs is a consequence of comparing to an incorrect reference power spectrum with high $\Omega_{M}$. The true value of $\Omega_{M}$ is lower, and the excess power is on small scales, as in Figure 12. The observed clustering was calculated using a weighted average over all galaxy pairs. Consequently, if we analyze the DR5 LRGs and main galaxies separately and match the largescale clustering amplitudes, we would expect a progression from a lower small-scale amplitude of the main galaxies, through the combined sample, to a higher clustering amplitude of the LRGs. Figure 19 shows that this is indeed what we find.

The combination of scale-dependent bias, redshift-space distortions, and nonlinear structure growth for a set of galaxies has previously been matched by simple fitting formulae (the $Q$-model) applied after calculation of the power spectrum (Cole et al. 2005). However, it remains to be seen if this simple prescription can adequately describe galaxy bias for LRGs or for a mixed sample of galaxies with different clustering properties; these issues will be considered in Tegmark et al. (2006). The analysis presented in our paper suggests that this correction could become increasingly important for samples as the average $r$-band galaxy luminosity increases. It is clear that, for LRGs, the relation between galaxies and dark matter will need to be carefully modeled in order to extract the maximum possible information from the shape of the observed power spectrum.

\section{SUMMARY}

In summary, using the SDSS, the redshift-space galaxy power spectrum is now known with sufficient accuracy on large scales to test the link between galaxies and the underlying matter distribution within the class of CDM models. If we assume a scaleindependent bias between galaxies and the mass, we find that no linear CDM model can fit the data over $0.01 h \mathrm{Mpc}^{-1}<k<$ $0.15 h \mathrm{Mpc}^{-1}$, with fits to $0.01 h \mathrm{Mpc}^{-1}<k<0.06 h \mathrm{Mpc}^{-1}$ suggesting a matter density that is $2-3 \sigma$ from that derived fitting to $0.01 h \mathrm{Mpc}^{-1}<k<0.15 h \mathrm{Mpc}^{-1}$. Perhaps the simplest explanation is that the large-scale distribution of LRGs is affected by scale-dependent bias, although we cannot rule out the alternative possibility that this is due to a systematic effect. If we calculate power spectra for subsets of galaxies selected using the $r$-band luminosity, then we only see weak evidence for a change in shape of the power spectrum on the appropriate scales, with galaxies that are less luminous having a scale-dependent bias that is weaker and affects smaller scales than more luminous galaxies. Such a bias model could also explain the bimodality in matter densities calculated from other data sets and from previous SDSS power spectra. The blue-selected 2dFGRS galaxies would be less affected by this scale-dependent bias than the redselected galaxies and bias modeling would be less important when calculating cosmological constraints. Hence, the simple assumption of Percival et al. (2001) that the 2dFGRS galaxy power spectrum has the same shape for $k<0.15 h \mathrm{Mpc}^{-1}$ as the matter power spectrum is comparatively harmless. As the $r$-band luminosity of the sample increases, the nonlinear relation between the galaxies and dark matter needs to be carefully modeled when providing cosmological constraints from galaxy clustering even on relatively large scales $k<0.15 h \mathrm{Mpc}^{-1}$.

We thank the referee, Joss Bland-Hawthorn, for helpful comments and suggestions. W. J. P. is grateful for support from a PPARC fellowship, and R. C. N. for a EU Marie Curie Excellence Chair.

Funding for the SDSS and SDSS-II has been provided by the Alfred P. Sloan Foundation, the Participating Institutions, the National Science Foundation, the US Department of Energy, the National Aeronautics and Space Administration, the Japanese Monbukagakusho, the Max-Planck Society, and the Higher Education Funding Council for England. The SDSS Web site is http://www.sdss.org.

The SDSS is managed by the Astrophysical Research Consortium for the Participating Institutions. The Participating Institutions are the American Museum of Natural History, Astrophysical Institute Potsdam, University of Basel, Cambridge University, Case Western Reserve University, University of Chicago, Drexel University, Fermilab, the Institute for Advanced Study, the Japan Participation Group, Johns Hopkins University, the Joint Institute for Nuclear Astrophysics, the Kavli Institute for Particle Astrophysics and Cosmology, the Korean Scientist Group, the Chinese Academy of Sciences (LAMOST), Los Alamos National Laboratory, the Max-Planck-Institute for Astronomy (MPIA), the MaxPlanck-Institute for Astrophysics (MPA), New Mexico State University, Ohio State University, University of Pittsburgh, University of Portsmouth, Princeton University, the United States Naval Observatory, and the University of Washington.

Simulated catalogs were calculated and analyzed using the COSMOS Altix 3700 supercomputer, a UK-CCC facility supported by HEFCE and PPARC in cooperation with CGI/Intel.
Abazajian, K., et al. 2003, AJ, 126, 2081 2004, AJ, 128, 502

Adelman-McCarthy, J., et al. 2006, ApJS, 162, 38

Baldry, I. K., Glazebrook, K., Brinkmann, J., Ivezic, Z., Lupton, R. H., Nichol, R. C., \& Szalay, A. S. 2004, ApJ, 600, 681

Bardeen, J. M., Bond, J. R., Kaiser, N., \& Szalay, A. S. 1986, ApJ, 304, 15

Baugh, C., \& Efstathiou, G. 1993, MNRAS, 265, 145

Blake, C., Collister, A., Bridle, S., \& Lahav, O. 2007, MNRAS, in press (astro$\mathrm{ph} / 0605303$ )

Blanton, M. R., Lin, H., Lupton, R. H., Maley, F. M., Young, N., Zehavi, I., \& Loveday, J. 2003a, AJ, 125, 2276

Blanton, M. R., et al. 2003b, ApJ, 592, 819 2005, AJ, 129, 2562

Bond, J. R., \& Efstathiou, G. 1984, ApJ, 285, L45 1987, MNRAS, 226, 655

Cole, S., et al. 2005, MNRAS, 362, 505

Coles, P., \& Jones, B. 1991, MNRAS, 248, 1

Colless, M., et al. 2001, MNRAS, 328, 1039 2003 , preprint (astro-ph/0306581)

Connolly, A., et al. 2002, ApJ, 579, 42

\section{REFERENCES}

Cooray, A., \& Sheth, R. 2002, Phys. Rep., 372, 1

Dodelson, S., et al. 2002, ApJ, 572, 140

Efstathiou, G., \& Bond, J. R. 1999, MNRAS, 304, 75

Efstathiou, G., \& Moody, S. J. 2001, MNRAS, 325, 1603

Efstathiou, G., Sutherland, W. J., \& Maddox, S. J. 1990, Nature, 348, 705

Eisenstein, D. J., \& Hu, W. 1998, ApJ, 496, 605

Eisenstein, D. J., Hu, W., \& Tegmark, M. 1999, ApJ, 518, 2

Eisenstein, D. J., Seo, H.-J., \& White, M. 2006, ApJ, submitted (astro-ph/ 0604361)

Eisenstein, D. J., et al. 2001, AJ, 122, 2267

2005, ApJ, 633, 560

Feldman, H. A., Kaiser, N., \& Peacock, J. A. 1994, ApJ, 426, 23

Fukugita, M., Ichikawa, T., Gunn, J. E., Doi, M., Shimasaku, K., \& Schneider, D. P. 1996, AJ, 111, 1748

Górski, K. M., Hivon, E., Banday, A. J., Wandelt, B. D., Hansen, F. K., Reinecke, M.,

\& Bartelmann, M. 2005, ApJ, 622, 759

Gott, J. R., et al. 2005, ApJ, 624, 463

Gunn, J. E., et al. 1998, AJ, 116, 3040

2006, AJ, 131, 2332

Hinshaw, G., et al. 2006, ApJ, submitted (astro-ph/0603451) 
Hogg, D. W., Finkbeiner, D. P., Schlegel, D. J., \& Gunn, J. E. 2001, AJ, 122, 2129

Holtzman, J. A. 1989, ApJS, 71, 1

Huetsi, G. 2006, A\&A, 449, 891

Ivezic, Z., et al. 2004, Astron. Nachr., 325, 583

Kaiser, N. 1987, MNRAS, 227, 1

Lupton, R., Gunn, J. E., Ivezic, Z., Knapp, G. R., Kent, S., \& Yasuda, N. 2001, in ASP Conf. Ser. 238, Astronomical Data Analysis Software and Systems X, ed. F. R. Harnden Jr., F. A. Primini, \& H. E. Payne (San Francisco: ASP), 269 Lupton, R. H., Gunn, J. E., \& Szalay, A. S. 1999, AJ, 118, 1406

Maddox, S. J., Efstathiou, G., \& Sutherland, W. J. 1996, MNRAS, 283, 1227

Maddox, S. J., Efstathiou, G., Sutherland, W. J., \& Loveday, J. 1990, MNRAS, 243, 692

Norberg, P., et al. 2001, MNRAS, 328, 64 2002, MNRAS, 332, 827

Padilla, N. D., \& Baugh, C. M. 2003, MNRAS, 343, 796

Padmanabhan, N., et al. 2006, MNRAS, submitted (astro-ph/0605302)

Page, L., et al. 2006, ApJ, submitted (astro-ph/0603450)

Park, C., Vogeley, M. S., Geller, M. J., \& Huchra, J. P. 1994, ApJ, 431, 569

Peacock, J. A., \& Smith, R. E. 2000, MNRAS, 318, 1144

Peebles, P. J. E., \& Yu, J. T. 1970, ApJ, 162, 815

Percival, W. J., \& Brown, M. L. 2006, MNRAS, 372, 1104

Percival, W. J., Verde, L., \& Peacock, J. A. 2004, MNRAS, 347, 645

Percival, W. J., et al. 2001, MNRAS, 327, 1297

$$
\text { 2002, MNRAS, 337, } 1068
$$$$
\text { 2007, ApJ, 657, } 51
$$

Pier, J. R., et al. 2003, AJ, 125, 1559

Pope, A. C., et al. 2004, ApJ, 607, 655

Press, W. H., Teukolsky, S. A., Vetterling, W. T., \& Flannery, B. P. 1992, Numerical Recipes in C: The Art of Scientific Computing (2nd ed.; Cambridge: Cambridge Univ. Press)
Saunders, W., et al. 2000, MNRAS, 317, 55

Schlegel, D. J., Finkbeiner, D. P., \& Davis, M. 1998, ApJ, 500, 525

Scranton, R., et al. 2002, ApJ, 579, 48

Seljak, U. 2000, MNRAS, 318, 203 2001, MNRAS, 325, 1359

Shectman, S. A., Landy, S. D., Oemler, A., Tucker, D. L., Lin, H., Kirshner, R. P., \& Schechter, P. L. 1996, ApJ, 470, 172

Silk, J. 1968, ApJ, 151, 459

Smith, J. A., et al. 2002, AJ, 123, 2121

Smith, R. E., et al. 2003, MNRAS, 341, 1311

Spergel, D. N., et al. 2003, ApJS, 148, 175 2006, ApJ, submitted (astro-ph/0603449)

Stoughton, C., et al. 2002, AJ, 123, 485

Strateva, I., et al. 2001, AJ, 122, 1861

Strauss, M. A., et al. 2002, AJ, 124, 1810

Sunyaev, R. A., \& Zel'dovich, Ya. B. 1970, Ap\&SS, 7, 3

Szalay, A., et al. 2003, ApJ, 591, 1

Tadros, H., et al. 1999, MNRAS, 305, 527

Tegmark, M., Taylor, A. N., \& Heavens, A. F. 1997, ApJ, 480, 22

Tegmark, M., et al. 2002, ApJ, 571, 191 2004, ApJ, 606, 702 2006, Phys. Rev. D, 74, 123507

Tucker, D., et al. 2006, Astron. Nachr., 327, 821

Vogeley, M. S., Park, C., Geller, M. J., \& Huchra, J. P. 1992, ApJ, 391, L5

Vogeley, M. S., \& Szalay, A. S. 1996, ApJ, 465, 34

Yahata, K., et al. 2006, PASJ, submitted (astro-ph/0607098)

York, D. G., et al. 2000, AJ, 120, 1579

Zehavi, I., et al. 2002, ApJ, 571, 172 2005a, ApJ, 621, 22

2005b, ApJ, 630, 1 